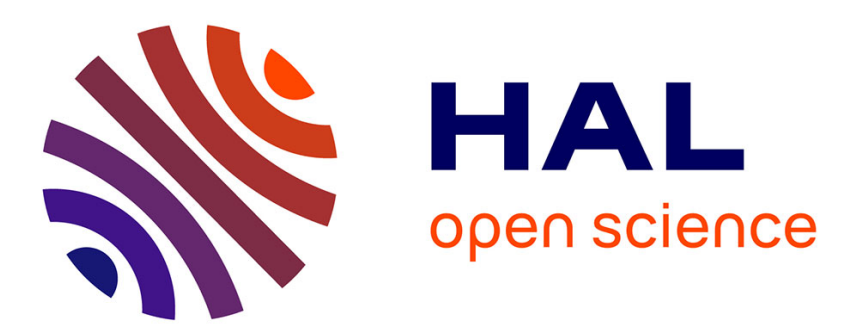

\title{
Non-Tuberculous Mycobacterial infections of veterinary relevance
}

\author{
Franck Biet, Maria-Laura Boschiroli
}

\section{To cite this version:}

Franck Biet, Maria-Laura Boschiroli. Non-Tuberculous Mycobacterial infections of veterinary relevance. European Symposium on Non-tuberculous Mycobacteria, Jun 2015, Borstel, Germany. 2015. hal-02742444

\section{HAL Id: hal-02742444 \\ https://hal.inrae.fr/hal-02742444}

Submitted on 3 Jun 2020

HAL is a multi-disciplinary open access archive for the deposit and dissemination of scientific research documents, whether they are published or not. The documents may come from teaching and research institutions in France or abroad, or from public or private research centers.
L'archive ouverte pluridisciplinaire HAL, est destinée au dépôt et à la diffusion de documents scientifiques de niveau recherche, publiés ou non, émanant des établissements d'enseignement et de recherche français ou étrangers, des laboratoires publics ou privés. 


\section{European Symposium on Non-tuberculous Mycobacteria}

24th - 27th June 2015

Manor House, Research Center Borstel

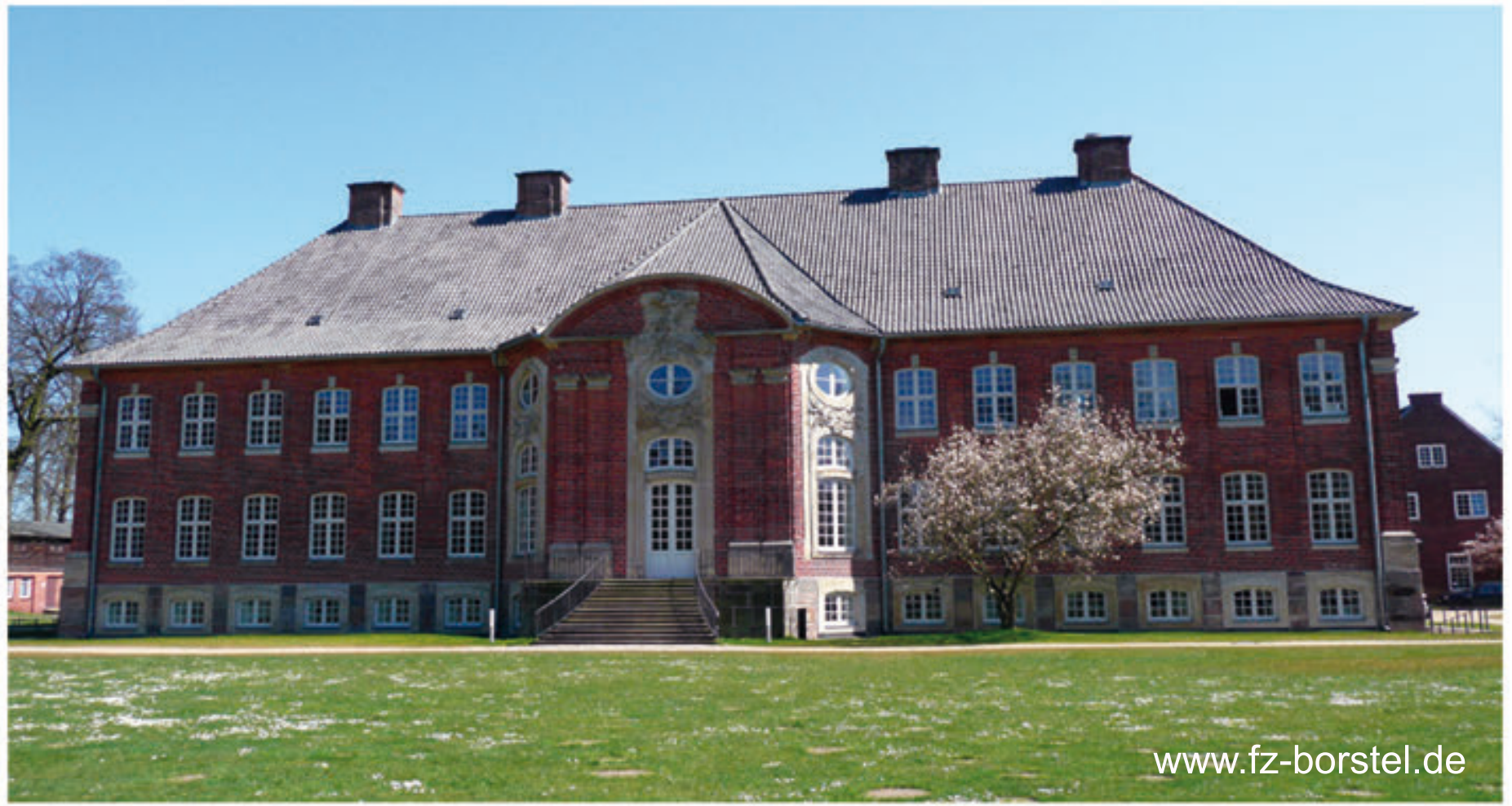

\section{Keynote Speakers}

Michel Arthur (Paris, France) · Kenneth Bruce (London, UK) · Andres Floto (Cambridge, UK) Jean-Louis Herrmann (Paris, France) - Jakko van Ingen (Nijmegen, The Netherlands) Laurent Kremer (Montpellier, France) · Kenneth Olivier (Bethesda, MD, USA)

Diane Ordway (Fort Collins, CO, USA) · Richard Wallace (Tyler, TX, USA)

\section{Scientific Advisory Board}

Andres Floto (Cambridge, UK) - Jean-Louis Herrmann (Paris, France) Jakko van Ingen (Nijmegen, The Netherlands) · Laurent Kremer (Montpellier, France) Ulrich Schaible (Borstel, Germany) · Dirk Wagner (Freiburg, Germany)

\section{Local Organizing Committee:}

Christian Herzmann · Sven Müller-Loennies · Jutta Passarger · Bianca Schneider Nasstaja Wassilew · Otto Holst (Chair) 



\section{European Symposium on Non-Tuberculous Mycobacteria}

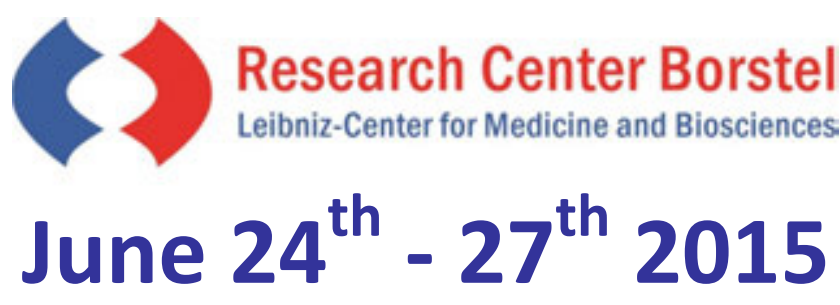

Scientific Advisory Board

Andres Floto (Cambridge, UK)

Jean-Louis Herrmann (Paris, France)

Jakko van Ingen (Nijmegen, The Netherlands)

Laurent Kremer (Montpellier, France)

Ulrich Schaible (Borstel, Germany)

Dirk Wagner (Freiburg, Germany)

\section{Local Organizing Committee}

Christian Herzmann

Sven Müller-Loennies

Jutta Passarger

Bianca Schneider

Nasstasja Wassilew

Otto Holst (Chair)

\section{Financially Supported By}
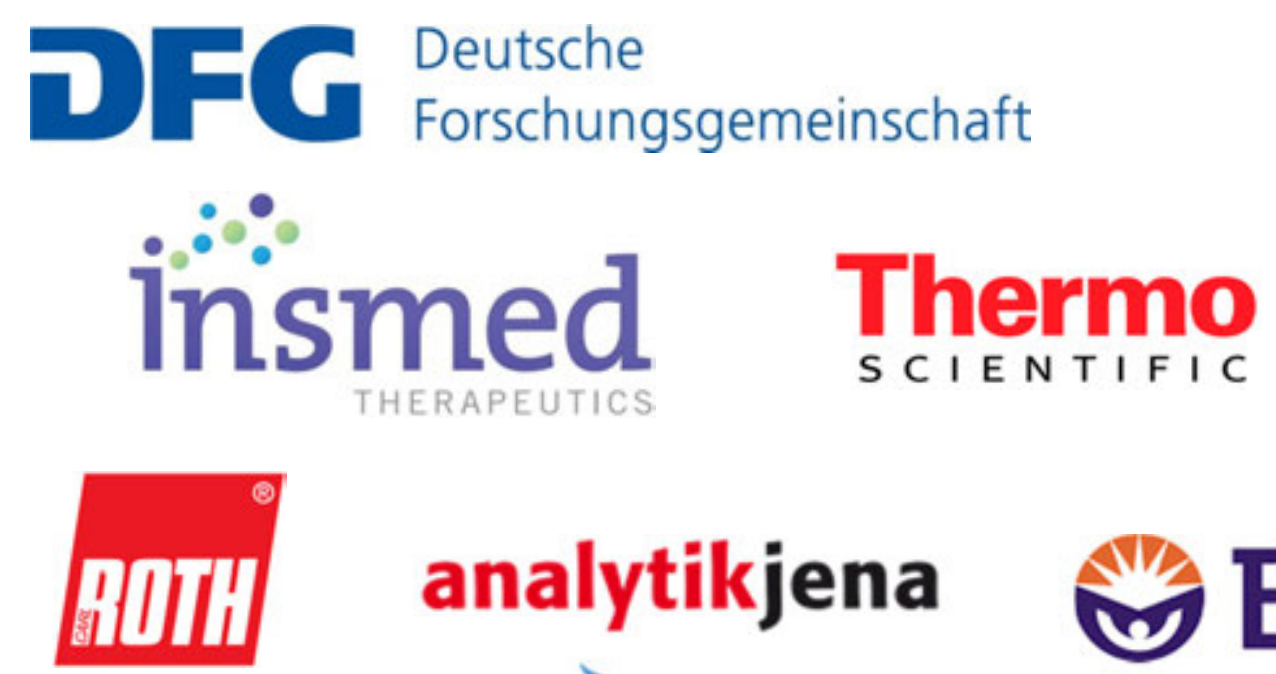

\section{analytikjena}

janssen

PHARMACEUTICAL COMPANIES

of Gohmon-gohmon

\section{Veiap}




\section{Program}

June $24^{\text {th }}, 2015$

16:00 - 18:00 Registration

18:00-21:30 Get together

\section{June $25^{\text {th }}, 2015$}

08:50

Welcome Address

Chair

Patrick Brennan, Fort Collins, USA

09:00 - 09:45 Kenneth Olivier, Bethesda, MD, USA

Evolving Geographic and Ecologic Patterns of NTM

09:45 - 10:05 Torsten Eckstein, Fort Collins, CO, USA

Phylogenetic characterization of the five subspecies of Mycobacterium avium, $M$.

intracellulare, and M. scrofulaceum

10:05 - 10:25 Walter Oelemann, Rio de Janeiro, Brazil

Comparison of lipid profiles from Mycobacterium paratuberculosis (MAP) isolated from the infected host and after subculture in vitro

10:25 - 10:55 Coffee Break

Chair Dirk Wagner, Freiburg i. Br., Germany

10:55 - 11:40 Diane Ordway, Fort Collins, CO, USA

Understanding non-tuberculous mycobacteria: pathogenesis and treatment

11:40 - 12:00 Dariimaa Ganbat, Borstel, Germany

Functional studies of the initial phase of mycobacterial infection in human lung tissues

12:00 - 12:20 Andrea Sanchini, Berlin, Germany

Mycobacterium avium subsp. hominissuis isolated in Germany: characterization of a new genomic island

12:30-14:00 Lunch 
14:00 - 14:45 Richard Wallace, Tyler, TX, USA

Mycobacterium avium lung disease and the modern day broad street pump: a tale of two cities

14:45 - 15:05 Franck Biet, Tours, France

Feature of adhesions expressed in Mycobacterium intracellulare

15:05 - 15:25 Fabienne Misguich, Montigny-le-Bretooneux, France

Type VII secretion pathway involved in Mycobacterium abscessus intracellular survival

15:25- 15:55 Coffee

Chair Ulrich Schaible, Borstel, Germany

16:00 - 16:45 Laurent Kremer, Montpellier, France

Deciphering and imaging the physiopathological events of Mycobacterium abscessus infection in zebrafish embryos

16:45 - 17:30 Jean-Louis Herrmann,

Mycobacterium abscessus: a peculiar behavior for a rapid growing mycobacterium inside cells

Dinner

\section{June $26^{\text {th }}, 2015$}

Chair

09:00-09:45

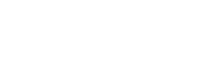

09:45 - 10:05

$10: 05-10: 25$

$10: 25-10: 55$

Faustine Dubar, Lille, France

Jakko van Ingen, Nijmegen, Netherlands

Assessing drug susceptibility of non-tuberculous mycobacteria in the clinical and pre-clinical setting

Sanne Zweijpfenning, Nijmegen, The Netherlands

Characteristics and treatment outcomes of non-tuberculous mycobacterial pulmonary disease in mycobacterial disease reference clinic

Caroline Demangel, Paris, France

Systemic immune suppression imposed by mycolactone: implications for the treatment of Buruli ulcer disease 
10:55 - 11:40 Michel Arthur, Paris, France

Mycobacteria and betalactams: mode of action and therapeutic perspectives

11:40 - 12:00 Heike Köhler, Jena, Germany

Comparative analysis of systemic and local host responses against MAP and MAH in an experimental goat model

12:00 - 12:20 Kris Huygen, Brussels, Belgium

Analysis of the virulence and immunogenicity of porcine and human M. avium ssp. hominissuis isolates in an experimental mouse model

12:30 - 14:00 Lunch

Chair

Brendan Loftus, Dublin, Ireland

14:00 - 14:45 Andres Floto, Cambridge, UK

Using whole genome sequencing to uncover the biology of NTM infection

14:45 - 15:30 Kenneth Bruce, London, UK

Cystic fibrosis airways microbiota - from ecology to treatment

15:30 - 18:00 Coffee and Poster

18:00

Dinner

June $27^{\text {th }}, 2015$

Departure 


\section{Abstracts}

Keynotes and Oral Presentations 


\title{
Evolving geographic and gcologic patterns of NTM
}

\author{
$\underline{\text { Kenneth N Olivier, MD, MPH }}$
}

National Heart, Lung, and Blood Institute/NIH

Bethesda, MD, USA

Reports from around the world note a similar awareness and often increasing prevalence of nontuberculous mycobacteria (NTM), particularly in association with lung disease. Population based studies have been limited by lack of reporting requirements for NTM, overlap of signs and symptoms of disease with other causes especially in TB endemic areas, the need for appropriate radiologic and culture based methods for detection, and a lack of access to complete information for proper disease characterization. In studies that have looked at serial changes in prevalence, there appear to be a consistent increase across disparate geographical areas. Quite a bit of variability has been noted with regard to relative prevalence of individual species of NTM. In general, many studies note a predominance of species within the $M$. avium complex, with the rapid grower mycobacteria such as $M$. abscessus being next most common followed by $M$. xenopi.

Historically, NTM have been associated with neoplasms and structural lung disease such as bronchiectasis and chronic obstructive pulmonary disease. Recent case control studies have supported these associations as well as factors such as thoracic skeletal abnormalities and low body mass index likely reflective of the "Lady Windermere" population of predominantly women without identified predisposing disease risks. The use of immunomodulatory drugs including steroids or autoimmune conditions such as rheumatoid arthritis have also been associated with NTM pulmonary disease. Regional differences in host characteristics have been described with nonsmoking women with idiopathic bronchiectasis predominating in the US and smoking men with COPD/emphysema more common in Europe.

In addition to host factor associations, there have been a number of recent reports of potential geographic and environmental risks with marked regional variations in prevalence for countries like the US, Japan, and Australia which may relate to differences in atmospheric, soil, or water conditions.

In summary, recent reports indicate an increasing prevalence of NTM pulmonary disease. It is likely a combination of host characteristics and environmental factors which influence disease occurrence. Marked regional variation is noted with regarding to both host characteristics and predominant species of NTM associated with disease. 


\title{
Phylogenetic characterization of the five subspecies of Mycobacterium avium, M. intracellulare, and M. scrofulaceum
}

\author{
Torsten M. Eckstein and Julia M. Inamine
}

Colorado State University, Department of Microbiology, Immunology, and Pathology, Fort Collins, CO 80523, USA

For a long time the $M$. avium complex consisted of three bacterial species: Mycobacterium avium, M. intracellulare, and $M$. scrofulaceum. All three mycobacterial species are known for their highly immunogenic surface glycopeptidolipids generating 28 different serovars for $M$. avium/intracellulare and one for $M$. scrofulaceum. Twenty-five years ago, $M$. paratuberculosis and $M$. silvaticum were reassigned as subspecies of $M$. avium resulting in three subspecies: subsp. avium, subsp. paratuberculosis, and subsp. silvaticum. Recently, it was demonstrated that the subsp. avium should be divided into two subspecies: one for bird isolates (subsp. avium) and one for non-bird isolates (subsp. hominissuis).

With the large amount of variation within this complex and new species identified that might be closely related to the $M$. avium complex it is necessary to assign the various serovars and subspecies to its correct bacterial species. Here we present our 16S rRNA gene sequence analyses to assign the various serovars to its original bacterial species and demonstrating that all subspecies recently designed belong to $M$. avium. We could show that all subspecies do far declared are subspecies of $M$. avium. We also could demonstrate that there are clusters for $M$. avium, $M$. intracellulare, and $M$. scrofulaceum. In addition, we identified another cluster outside the three clusters. Serovars $1-6,9-11$, and 21 are M. avium. Serovars $12-17,19,20$, and 25 are $M$. intracellulare. Serovar 7 is split between $M$. intracellulare (2 strains) and $M$. scrofulaceum (2 strains). Serovar 8 can be $M$. avium or $M$. intracellulare. Serovar 18 was found in the cluster of $M$. intracellulare and in the cluster of $M$. scrofulaceum. Serovar 22 was seen within the $M$. intracellulare cluster and in the outside cluster. Further studies on the actual carbohydrate composition and reassurance of the serovar determination of the serovars with split assignments are needed. 


\title{
Comparison of lipid profiles from Mycobacterium paratuberculosis (MAP) isolated from the infected host and after subculture in vitro
}

\author{
Walter M. R. Oelemann ${ }^{1}$, Matthias Stehr ${ }^{2}$, Regina Engel ${ }^{3}$, Katarzyna A. Duda ${ }^{3}$ \\ and Ralph Goethe ${ }^{2}$
}

\begin{abstract}
${ }^{1}$ Departamento de Imunologia, Instituto de Microbiologia, Universidade Federal do Rio de Janeiro (UFRJ), Rio de Janeiro, Brazil, oelemann@micro.ufri.br

${ }^{2}$ Department of Infectious Diseases, Institute for Microbiology, University of Veterinary

Medicine Hannover, Hannover, Germany

${ }^{3}$ Division of Structural Biochemistry, Research Center Borstel, Leibniz-Center for Medicine and Biosciences, Borstel, Germany
\end{abstract}

Mycobacterium avium ssp. paratuberculosis (MAP) is the etiological agent of Johne's disease or paratuberculosis. MAP is able to infect monogastric animal species and humans. Similar to other pathogenic mycobacteria, the highly complex lipid repertoire of the cell wall contributes to host-pathogen interaction and bacterial survival in the host. In the host, mycobacteria modify significantly their lipid metabolism, and during the course of the resulting long-term infection, mycobacterial lipids seem to constantly influence the immune response of the host. Some reports suggest that the lipid pattern changes from more polar molecules in the beginning of an infection to more apolar compounds during later stages. In the present work we compare the lipid profiles of MAP isolated by sucrose gradient centrifugation from the intestinal mucosa of an infected cow and the same isolate harvested after in vitro surface culture on Middlebrook medium supplemented with mycobactin. Both bacterial preparations were lyophilized and non-covalently bound lipids were extracted by thorough stirring in successive mixtures of chloroform/methanol in the proportions of 2:1, 1:1 and 1:2. The resulting crude lipids were fractionated on silica gel columns using a polarity gradient for elution. Lipid fractions were analyzed by one-dimensional and two-dimensional thin layer chromatography (TLC), using solvent systems which are suited for the different mycobacterial lipid classes (modified from Pirson et al. Vet. Res. 43:54 (2012)). These experiments indicate quantitative and qualitative differences in the lipid repertoire of MAP recovered from the gut and from the respective in vitro culture. The differences are predominantly observed in the fractions containing apolar lipids, as well as the polar fractions that contain phosphatidylinositol mannosides (PIMs). The PIM-containing fraction of MAP isolated from the gut appeared more complex than that of the corresponding isolate cultured in vitro. In contrast, MAP from culture appeared to synthesize more apolar lipids. Presently, the fractions of interest are being separated/purified utilizing HPLC withreverse phase column. The obtained fractions will be chemically characterized by GC/MS and ESI MS. 


\section{Understanding non-tuberculosis mycobacteria: pathogenesis and treatment}

\footnotetext{
Diane Ordway ${ }^{1}$, Kimberly Arnett ${ }^{1}$, Deepshikha Verma ${ }^{1}$, Megan Blackledge $^{2}$, Christian Melander $^{2}$, Anne Lenaerts ${ }^{1}$

${ }^{1}$ Colorado State University, D.Ordway@colostate.edu

${ }^{2}$ North Carolina State University

Over the past decade there has been a significant increase in cases of drug resistance associated with nontuberculous mycobacterial (NTM) species. Little is known about the nature of the pathogenesis of NTM infections and how these organisms overcome host immunity and persist in vivo. This explains the current lack of development of more efficacious and novel targeted therapies. The cure rate for chronic lung and disseminated infections due to NTM is dismal despite prolonged treatment with various multi-drug regimens. Mycobacterium abscessus outcomes are much worse than other rapidly growing mycobacteria (RGM), in fact this organism seems to be essentially untreatable, with only $\sim 50 \%$ sputum conversion seen in multiple chemotherapy studies. Incorrect drug susceptibility predictions - combined with minimal pharmacokinetics and pharmacodynamics information for current treatment regimens-has led to these pathogens being associated with alarming high fatality rates. Our preliminary results with animal models of NTM infection support the hypothesis that these NTMs are capable of using different routes of infection resulting in biofilm formation and phenotypic antimicrobial resistance. Our studies provide strong evidence that through inhibition of biofilm formation with small molecule inhibitors during in vitro/in vivo infection with $M$. abscessus increased bactericidal activity is present when combined with the standard anti-NTM treatment. Moreover, additional approaches using adjuvant inhibitors combinated with immunomodulatory agents show promise towards NTM prevention.
} 


\title{
Functional studies on the initial phase of mycobacterial infection in human lung tissues
}

\author{
Dariimaa Ganbat ${ }^{1}$, Elvira Richter ${ }^{3}$, Sebastian Marwitz ${ }^{2}$, Ekkehard Vollmer $^{2}$, Christian Kugler ${ }^{4}$, \\ Torsten Goldmann ${ }^{2}$
}

\author{
${ }^{1}$ Mongolian National University of Medical Sciences, Ulaanbaatar, Mongolia (dganbat@fz- \\ borstel.de ) \\ ${ }^{2}$ Clinical and Experimental Pathology, Research Center Borstel, Borstel, Germany \\ ${ }^{3}$ National Reference Centre for Mycobacteria, Forschungszentrum Borstel, Borstel,Germany \\ ${ }^{4}$ Hospital Großhansdorf , Thoracic surgery, Germany
}

Mycobacterial infections are still significant causes of morbidity and mortality. There are still surprising gaps in our knowledge about these infectious diseases because of limitations of available model systems. Particularly the early phase of mycobacterial infection remains unclear, whereas the late and active stages have been characterized by histological and clinical investigations. There are several lines of epidemiological evidences supporting that innate immunity might prevent or even eliminate mycobacterial infection. This attractive hypothesis and the limitations of human complex models for mycobacterial infection, the primary goal of the study was to establish a novel ex vivo STST (Short-Term Stimulation of Tissue) model for the initial phase of mycobacterial infection also allowing further functional studies.

From surgical material of 65 patients, $0.5-1 \mathrm{~cm}^{3}$ sizes of the human vital lung specimens were obtained and incubated with one of three different mycobacterial species $(M$. tuberculosis, $M$. avium, $M$. abscessus). The establishment of the ex vivo STST model was assessed by two main criteria: 1) viability of tissues, including histopathology, MTT and TUNEL assay; 2) infection of tissues, including histo and molecular pathological methods.

The morphologic comparisons between ex vivo incubated and non-incubated lung specimens did not show any noticeable differences. The MTT assay showed that the optical measurements of the ex vivo incubated samples (2.34 \pm 0.12$)$ intervened between positive $(3.52 \pm 0.06)$ and negative controls $(0.22 \pm 0.01)$. By a TUNEL assay we determined that $92.85 \pm 6.99 \%$ of cells in the ex vivo stimulated tissues were alive. Serial sections of a whole block verified that diffusion of infectious agents deep into tissues was sufficient. Genetic material of mycobacteria could be detected in the stimulated tissues. We observed that different cell types were infected, including macrophages, neutrophils, monocytes and pneumocytes-II. A total of 900 infected cells were counted and macrophages and monocytes predominated $(76.58 \%$ and $3.03 \%)$ regardless of the mycobacterial strain ( $p=0.539$ and $\mathrm{p}=0.761$ ). Overall incidences of infected neutrophils was $7.46 \%$, infection of pneumocytes-II exhibited $12.93 \%$. Moreover, neutrophils were predominantly infected by $M$. abscessus, whereas infected pneumocytes-II were more commonly found after infection within $M$. tuberculosis. Infected cells also responded with cellular changes, including karyopyknosis, karyrrhexis, and karyolysis depending on the mycobacterial species. Transcriptome analysis showed that in sets of $M$. abscessus 840, M. tuberculosis 1018, M. avium 5944 genes were up-regulated upon stimulation compared to medium control.

Tissue viabilities were acceptable and infections of the tissues were successful. All collected patients' lung specimens were infected and showed that the initial host defense mechanism is actively working via phagocytosis without any additional stimulation, unlike in vitro experiments. The combination of short-term stimulated vital lung specimens and HOPE fixation technology allow the extraction of high quality mRNA and a genome-wide expression profile upon mycobacterial infection can be investigated on mRNA level. 


\title{
Mycobacterium avium subsp. hominissuis isolated in Germany: characterization of a new Genomic island
}

\author{
$\underline{\text { Andrea Sanchini }}{ }^{1}$, Annesha Lahiri ${ }^{1}$, Torsten Semmler ${ }^{2}$, Inga Eichhorn ${ }^{2}$, Niyaz Ahmed ${ }^{3}$, \\ Narender Kumar ${ }^{3}$, Kshitij Tandon ${ }^{3}$, Astrid Lewin ${ }^{1}$ \\ ${ }^{1}$ Division of Mycotic and Parasitic Agents and Mycobacteria (FG16), Robert Koch Institut, \\ Berlin; sanchinia@rki.de \\ ${ }^{2}$ Freie Universität Berlin, Centre for Infection Medicine, Institute of Microbiology and \\ Epizootics, Berlin, Germany \\ ${ }^{3}$ Pathogen Biology Laboratory, Department of Biotechnology and Bioinformatics, University \\ of Hyderabad, India
}

Incidences of infections caused by Non-tuberculous mycobacteria (NTM) are increasing worldwide. The specie Mycobacterium avium is a one of the most relevant among the group of NTM. Within the $M$. avium specie, the subspecies $M$. avium subsp. hominissuis (MAH) is an opportunistic human pathogen causing lymphadenitis, lung, skin and disseminated infections. It is also widespread in the environment, especially dust, soil and water. Genomic Islands (Gl)s are mobile genetic elements that can be involved in pathogenesis, fitness, metabolism, or drug-resistance. Previous work identified a new Gl in $4 \mathrm{MAH}$ genomes available in GenBank. Here we identify such GI in environmental and clinical strains of MAH isolated in Germany and we characterize the Gl gene composition.

A total of $50 \mathrm{MAH}$ strains were analyzed. Genomic DNA has been extracted by Phenol/Chloroform extraction. Whole genome sequencing has been performed using Illumina MiSeq Desktop Sequencer. Reads were assembled using CLC Genomics Workbench in form of contigs. Contigs were annotated using RAST. The conserved flanking genes present on either sides of the Gl were used for the identification of the Gls in the contigs. All genes of the Gis have been extracted using Geneious. The 28 most frequent genes of the Gls which have hypothetical annotation have been analyzed by I-Tasser, SWISS modeling and DALI server in order to putatively assign protein function.

In $18 / 50$ strains (36\%) a complete GI has been identified. In $2 / 50$ strains (4\%) no GI has been identified. In $30 / 50$ strains $(60 \%)$ the $\mathrm{Gl}$ is present but it is not fully covered by the assembled sequences, suggesting sequence variability and the needs of further assembly methods. The most common $\mathrm{Gl}$ is of $16.4 \mathrm{~kb}$. The size of the $\mathrm{Gl}$ (from 13,4 to $84,9 \mathrm{~kb}$ ) and the gene composition varies across the strains. The Gl of some strains carries a homolog of the mmpl10 gene (involved in other mycobacteria in lipids transport and cell wall biosynthesis). Computational biology of genes annotated as suggests that it may be involved in drug-resistance. Computational biology of genes with hypothetical protein annotation revealed that $8 / 17(47 \%)$ proteins may have a function in transcription regulator. $7 / 17(41 \%)$ proteins may have enzymatic activity $2 / 17(12 \%)$ proteins may act as fibrinogen-binding protein.

The recently identified GI is present also in a German collection of MAH strains isolated from various sources. Such GI is a region of high diversity. The Gl may carry genes potentially interesting for drug-resistance (e.g. mmp/10). Generation of knock-out mutants, gene up/down regulation and functional studies will assess if the Gl play a role in drug-resistance or host-pathogen interaction. 


\title{
Mycobacterium avium lung disease and the modern day broad street pump: a tale of two cities
}

\author{
Richard J. Wallace, Jr., Elena lakhiaeva, Barbara A. Brown- Elliott, Julie V. Philley, Jeana L. \\ Benwill, Rebecca R. Wilson, Sruthi Vasireddy, Joseph Falkinham III
}

Departments of Microbiology and Medicine, the University of Texas Health Center at Tyler, Tyler, TX, U.S.A.; Department of Biological Sciences, Virginia Tech, Blacksburg, VA, U.S.A. richard.wallace@uthct.edu

Little is known of the long term consequences of high risk patients to exposure to municipal water supplies containing multiple genotypes of Mycobacterium avium.

We identified 17 patients with NTM lung disease from two adjacent small southern U.S. towns (designated A and B) followed with serial sputum AFB cultures for 4-10 years. Patients were older, non-smoking white females and 17/18 had nodular bronchiectatic MAC lung disease. Town $A$ received its municipal water from a single purification plant processing surface water, while Town B received its water from an aquifer. MAC species were identified by sequencing and strain comparison by VNTR.

Approximately 400 sputum cultures (mean 45.3 per patient) were obtained from nine patients from Town A over a mean of 6 years. Of six sputum genotypes of $M$. avium identified, two were recovered from multiple patients (VNTR types 23 and 38). Multiple patients also had $M$. yongonense. 8/9 patients had $M$. avium type 38 over the mean 6 year period and 3/9 patients had all three genotypes at different times. Biofilms from household water systems in Town $A$ at the end of the study period revealed the same three epidemic MAC species/genotypes. Town B was less than one mile from Town A but as noted had a different water supply. Approximately 220 sputum cultures (mean 27.6 per patient) were obtained from eight patients in Town B over a mean of 5 years follow up. Only 2/8 patients had $M$. avium identified in their sputum, and none of the six $M$. avium genotypes or $M$. yongonense found in Town A were found in the sputum or household water of patients in Town B. Equivalent numbers of patients in both towns were infected with $M$. intracellulare, $M$. abscessus, and $M$. chimaera, but none of these species were recovered from household water.

This study implicates municipal water systems as the major source of $M$. avium nodular lung disease, and that the predominant $M$. avium biofilm genotypes can be acquired over many years from the same water source (hence the historical reference to the contaminated Broad Street pump described by Edward Snow as a source of cholera in London in the $19^{\text {th }}$ century). Thus patients with multiple genotypes of $M$. avium in their municipal water supply may in part explain the frequent recurrence of new genotypes of MAC in patients with nodular disease. This is the first study to evaluate longitudinal acquisition of MAC from individual communities and their relationship to household (municipal water systems). 


\title{
Feature of adhesins expressed in Mycobacterium intracellulare
}

\author{
Lefrancois $^{1}$, L. H., M. Branger ${ }^{1}$, T. Cochard ${ }^{1}$, O. Peuchant ${ }^{2}$, D. Raze ${ }^{3}$, C. Locht ${ }^{3}$, P. Lanotte ${ }^{1}$, \\ and F. Biet $^{1}$
}

1 INRA-Centre de Tours, UR1282, Infectiologie et Santé Publique (ISP-311), F-37380 Nouzilly, France. 2 Laboratoire de Bactériologie, Hôpital Haut-Lévèque-CHU de Bordeaux, Avenue Magellan, F-33604 Pessac France., 3 Inserm U1019, Lille France, CNRS UMR 8204, Lille, France, Institut Pasteur de Lille. Center for Infection and Immunity of Lille Lille, France, University Lille Nord de France, Lille, France, 4 Service Bactériologie-Virologie, Hôpital Bretonneau-CHRU de Tours, 2 boulevard Tonnellé, F-37044 Tours cedex France

\section{Franck.Biet@tours.inra.fr}

The Mycobacterium avium MAC complex includes two closely related species Mycobacterium avium and Mycobacterium intracellulare (Mi). These non-tuberculous mycobacteria are opportunistic pathogens in humans and responsible of severe diseases in a wide variety of animals. These bacteria are also isolated from various environmental sources. In humans $\mathrm{Mi}$ is the second cause of illness after the strains of Mycobacterium tuberculosis complex. The genomic data available for the reference strain ATCC 13950 have enabled the development of genotyping but few studies concern identification of factors involved in the pathogenicity of this mycobacteria.

The objective of this study was to identify, isolate and characterize the adhesins belonging to the family of HBHA and LBP from the reference strain and from a panel of clinical isolates from patients with different pathologies associated with Mi infection.

Based on the genomic sequence of Mi we investigated whether the genes of HBHA and LBP were present and conserved between the different strains of the panel. The expression of adhesin HBHA and LBP in Mi was investigated by Western blot from lysates of human isolates Mi. An approach using affinity chromatography on heparin Sepharose was used to isolate and purify native adhesins from Mi.

Our results allow for the first time the characterization of the expression of adhesins HBHA and LBP in Mi. This preliminary work opens numerous perspectives on the role of these molecules in human pathologies but also in diagnostic approaches of NTM infections in human. 


\title{
Type VII secretion pathway involved in Mycobacterium abscessus intracellular survival
}

\author{
Laencina Laura, Herrmann Jean-Louis, Misguich Fabienne
}

EA3647 EPIM Laboratory of Diagnostic and Physiopathology of Microbial Infection, U1173 INSERM Infection - Inflammation, UFR des Sciences de la Santé Simone Veil, Université de Versailles Saint-Quentin-en-Yvelines, Montigny-le-Bretonneux, France, fabienne.girardmisguich@uvsq.fr

The Mycobacterium genus brings together species ranging from harmless saprophytic organisms to major human pathogens. The well-known pathogenic species such as Mycobacterium tuberculosis, Mycobacterium marinum and Mycobacterium ulcerans belong to the subgroup of slowly growing mycobacteria (SGM). By contrast, rapidly growing mycobacteria (RGM) form a group of over 150 species essentially composed of nonpathogen saprophytic organisms. However, Mycobacterium abscessus, an RGM, was recently considered as an opportunistic human pathogen, responsible for a wide spectrum of infections, from pulmonary to skin and soft tissues infections. M. abscessus is considered, with Mycobacterium avium, as the major mycobacterial pathogen in patients with cystic fibrosis.

Mycobacterium abscessus has been shown to behave as an intracellular pathogen, able to survive to the bactericidal response of macrophages and fibroblasts in lungs and skin, survival usually not observed for RGM. Sequence analysis of $M$. abscessus genome showed that this bacterium is endowed with the metabolic pathways typically found in environmental micro-organisms with contact to soil, plants, and aquatic environments, where free-living amoeba are frequently present. Very little is known about the genetic requirements that $M$. abscessus has developed or acquired to survive in an intracellular environment. We aimed to find if $M$. abscessus possess genomics advantages to survive in antigen presenting cells such as macrophages or in environmental protozoa such as amoeba.

A transposon mutant library of a clinical strain of $M$. abscessus subspecies massiliense was screened by its ability to persist in amoeba and macrophages. Several mutants were identified to have a reduced ability to survive in those intracellular environments. Interestingly, twelve on 6,000 mutants screened appeared to be muted in genes homologs to the type VII secretion pathway of $M$. tuberculosis also present in M. abscessus. These twelve mutants were all in the ESX-4 locus. This evolutionary system of secretion could manipulate the host cell biology to make macrophages and amoeba attractive hosts for $M$. abscessus. We showed here for the first time, genes allowing $M$. abscessus to survive inside phagocytic cells present in the environment and in the human host. 


\title{
Deciphering and imaging the physiopathological events of Mycobacterium abscessus infection in zebrafish embryos
}

\author{
Laurent Kremer \\ Centre d'étude des Pathogènes pour la Biotechnologie et la santé (CPBS), CNRS FR3689, \\ University of Montpellier, 1919 route de Mende, 34293 Montpellier, France \\ Email: laurent.kremer@cpbs.cnrs.fr
}

The emerging pathogen Mycobacterium abscessus is a rapidly-growing mycobacterium causing severe lung infections, particularly in cystic fibrosis patients. The Smooth (S) morphotype expresses glycopeptidolipids (GPL) at the bacterial surface while the Rough (R) morphotype is characterized by the loss of surface GPL. Previous studies suggested that the $R$ variant is involved in more severe clinical forms, associated with a hyper-proinflammatory response. However, the molecular mechanisms responsible for the virulence associated to the $R$ variant remain elusive. The zebrafish embryo offers many advantages that motivated its use for a better understanding of mycobacterial infections.

We have developed a zebrafish model of infection to investigate and compare the pathogenesis of $R$ and $S$ variants. This model allows to describing and imaging the chronology of the infection by videomicroscopy. Both the antisense morpholino technology and zebrafish reporter lines were used to evaluate the contribution of the innate immune system. In contrast to the $S$ variant, the $R$ morphotype induced a more robust and lethal infection in embryos, characterized by the rapid development of bacterial foci within the Central Nervous System. The use of a mpeg1:mCherry transgenic zebrafish line, harbouring red fluorescent macrophages, demonstrated the presence of isolated or small aggregated bacilli within macrophages during early infection. Later stages were characterized by the presence of extracellularly-replicating mycobacteria, leading to rapid tissue damage (abscess) and to larval death. The high propensity of the $\mathrm{R}$ variant to form cords in vivo prevented the bacilli from being phagocytozed by macrophages or granulocytes, representing a mechanism of immune evasion. We also demonstrated the critical role of TNF signaling in controlling the infection of $S$ and $R$ variants. TNF receptor morphants were characterized by increased macrophage death, promoting uncontrolled extracellular growth of mycobacteria, and resulting in hyper-cording of the $R$ variant and enhanced larval death.

In addition, we propose to use this model to monitor, at spatiotemporal level, the in vivo therapeutic effects of molecules active against $M$. abscessus, notorious for being one of the most antibiotic-resistant mycobacterial species. 


\title{
Mycobacterium abscessus: a peculiar behavior for a rapid growing mycobacterium inside cells
}

\author{
Jean-Louis Herrmann
}

UMR1173 -Equipe EPIM, INSERM et UFR Des Sciences de la Santé Paris lle de France

Ouest, Université de Versailles - St Quentin en Yvelines, France

Mycobacterium abscessus is considered as an emerging pathogen in cystic fibrosis patients, with recent studies showing its genomic and phenotypic heterogeneity. Our main objective was to understand the particular sensitivity of cystic fibrosis patients, and more specifically of the CFTR $^{-1}$ macrophages and dendritic cells towards $M$. abscessus. We studied $M$. abscessus pathogenicity in vivo or ex vivo in mice or within macrophages and dendritic cells wild type, DF508, and $\mathrm{cftr}^{-1}$ respectively. By using in vivo/ex vivo animal and cellular models already developed in our laboratory, we were able to show the capacity of $M$. abscessus to resist to the intracellular bactericidal activity of macrophages, independently of the presence of a CFTR defect. 


\title{
Assessing drug susceptibility of non-tuberculous mycobacteria in the clinical and pre-clinical setting
}

\author{
Jakko van Ingen \\ Department of Medical Microbiology, Radboud University Medical Center, Nijmegen, the \\ Netherlands
}

Treatment of non-tuberculous mycobacterial (NTM) disease has developed along 2 lines; first, for slow growers such as Mycobacterium avium, drug regimens for tuberculosis have been modified to exclude pyrazinamide and include macrolides after their in vitro activity was shown. Yet, the use of rifampicin, ethambutol and streptomycin cannot be supported on basis on in vitro activity since these drugs have very little activity in vitro, both alone and in combinations. For the rapid growers, treatment regimens have been based on the speciesspecific in vitro susceptibilities. Yet, for some key drugs, such as aminoglycosides, the breakpoints to define susceptibility have largely been based on wild type minimum inhibitory concentration (MIC) distributions, even if these are concentrations impossible to achieve in serum or at the site of infection owing to toxicity. The recent insights in pharmacokinetics and pharmacodynamics of antimycobacterial drugs need to be used to redesign treatment regimens. The poor outcomes of currently recommended treatment regimens are testimony to the fact that these regimens need to be rebuilt from ground level.

In this presentation, correlations between in vitro susceptibility and in vivo treatment outcomes of major NTM diseases in trials and case series will be discussed. Then, an up-todate overview of results from recent time-kill kinetic assays performed for aminoglycosides, macrolides, linezolid, moxifloxacin and clofazimine against $M$. abscessus subsp abscessus, M. fortuitum, M. avium subsp hominissuis, M. xenopi and $M$. simiae wil be presented. Also, the first results of hollow fiber model experiments to determine the optimal dose and dosing strategy for amikacin against $M$. abscessus subsp abscessus will be presented. 


\title{
Characteristics and treatment outcomes of nontuberculous mycobacterial pulmonary disease in a mycobacterial disease reference clinic
}

\author{
Sanne Zweijpfenning ${ }^{1}$, Cecile Magis-Escurra ${ }^{1}$, Jakko van Ingen ${ }^{2}$, Martin Boeree ${ }^{1}$, \\ Wouter Hoefsloot ${ }^{1}$ \\ ${ }^{1}$ Radboud University Medical Center Nijmegen, Department of pulmonary diseases, PO Box 9101, 6500HB \\ Nijmegen, The Netherlands, email presenting author: sannezweijpfenning@gmail.com
}

The incidence of nontuberculous mycobacterial pulmonary disease (NTM-PD) is increasing in the last few years in the Netherlands. Evidence concerning treatment is poor. In this study we give an overview of clinical aspects, treatment and outcome of all patients with suspicion of clinical relevant NTM-PD referred to the center of expertise for mycobacterial infections (University lung center Dekkerswald, Nijmegen, The Netherlands).

We conducted a retrospective study of all patients seen between 2008 and 2013 with at least one NTM isolate and diagnosed using the American Thoracic Society (ATS) diagnostic criteria. Patients with non classical radiographic features that were not described in the ATScriteria were included as well.

334 patients were reviewed. Sixty-four patients with NTM-PD were included. Half of them were female and mean age was $64,2 \pm 12,3$ years. $M$. avium complex (MAC) was the predominant species ( $\mathrm{n}=38,59,3 \%) ; M$. malmoense and $M$. kansasii were seen in 7 and 6 patients. M.abcessus was seen in 4 patients, M.chimaera in 1 patient, M.simiae in 3 patients, M.xenopi in 2 patients and 3 patients had combined infections. The most predominant radiologic feature were nodules and consolidations $(60,9 \%)$. Most patients had underlying COPD (73,4\%), bronchiectasis was present in 54,7\%, 40,6\% smoked at time of diagnoses and $32,8 \%$ had a history of smoking. $87,8 \%$ of patients treated for MAC-PD were given a rifamycin-ethambutol-macrolide regimen. Five patients with MAC were not treated. From the patients who received rifamycin-ethambutol-macrolide regimen five patients additionally received clofazimine and 7 patients received amikacine and clofazamine. All six patients with pulmonary $M$. kansasii disease were treated with rifampicin and ethambutol; four also received isoniazid. The overall cure rate was $62,5 \%$; $66 \%$ for MAC, $66,7 \%$ for $M$. kansasii and $57,1 \%$ for $M$. malmoense. All-cause mortality during treatment was $20,3 \%$; ten patients experienced treatment failure $(=15,6 \%)$, and 7 patients, $10,9 \%$ relapsed.

In our setting, MAC was the predominant NTM and treatment largely followed ATS guidelines. Treatment success rates are comparable with data from the literature. The poor outcomes and toxicity underline the need for clinical trials addressing NTM-PD treatment. 


\section{Systemic immune suppression imposed by mycolactone: implications for the treatment of Buruli ulcer disease}

Laure Guenin-Macé $^{1,2}$, Ludivine Baron ${ }^{1,2}$, Anne-Caroline Chany ${ }^{4}$, Cédric Tresse ${ }^{4}$, Sarah Saint-Auret $^{4}$, Friederike Jönsson ${ }^{5,6}$, Jean-David Morel ${ }^{1,2}$, Sophie Hidalgo-Lucas ${ }^{7}$, JeanFrançois Bisson ${ }^{7}$, Nicolas Blanchard ${ }^{4}$, Caroline Demangel $^{1,2,3}$

${ }^{1}$ Institut Pasteur, Unité d'Immunobiologie de l'Infection, Paris, France

${ }^{2}$ CNRS URA 1961, Paris, France

${ }^{4}$ Université de Strasbourg, Laboratoire de Chimie Moléculaire, ECPM-CNRS UMR7509, France.

${ }^{5}$ Institut Pasteur, Unité Anticorps en Thérapie et Pathologie, Paris, France

${ }^{6}$ INSERM U760

${ }^{7}$ ETAP, Inflammation, Dermatologie et Toxicologie, Vandœuvre-lès-Nancy, France

The release of mycolactone by Mycobacterium ulcerans in host skin causes a combination of ulcerative, analgesic and anti-inflammatory effects. While ulcer formation is caused by the pro-apoptotic activity of mycolactone on skin cells via hyper-activation of Wiskott-Aldrich Syndrome proteins, analgesia results from neuronal hyper-polarization via signaling through angiotensin II type 2 receptors. Mycolactone also blunts the capacity of immune cells to produce inflammatory mediators, by an independent mechanism of protein synthesis blockade. In an attempt to isolate the structural determinants of mycolactone's immunosuppressive activity, we screened a library of synthetic subunits of mycolactone for inhibition of cytokine production by activated $T$ cells. The minimal structure retaining activity was a truncated version of mycolactone (hereafter called mini-mycolactone), missing one of its two polyketide chains. This compound inhibited the inflammatory cytokine responses of human primary cells at non-cytotoxic doses, and bound to angiotensin II type 2 receptors comparably to mycolactone in vitro. Intraperitoneally-delivered mini-mycolactone diffused rapidly into mouse peripheral blood cells. Moreover, like full mycolactone, it conferred systemic protection against chronic skin inflammation and inflammatory pain. In addition to identifying the structural basis of mycolactone bioactivity, our results establish its systemic effects against inflammation and pain. They highlight the need for continuous monitoring of mycolactone levels during the course of antibiotic therapy, in order to anticipate paradoxical reactions delaying the healing of Buruli ulcers. 


\section{Mycobacteria and beta-lactams: mode of action and therapeutic perspectives}

Michel Arthur ${ }^{1}$, Vincent Dubée ${ }^{1}$, Jean-Luc Mainardi ${ }^{1}$, Audrey Bernut ${ }^{2}$, Mélanie Cortes ${ }^{1}$, JeanEmmanuel Hugonnet ${ }^{1}$, Jean-Louis Hermann ${ }^{3}$, and Laurent Kremer $^{2}$

${ }^{1}$ Centre de Recherche des Cordeliers, LRMA Equipe 12. INSERM. Université Pierre et Marie Curie. Université Paris Descartes. Paris, France

${ }^{2}$ Mycobacterial Pathogenesis and Novel Therapeutic Targets. CNRS FRE368. Montpellier, France

${ }^{3}$ UMR1173 -Equipe EPIM. INSERM. Université de Versailles - St Quentin en Yvelines, France

The essential targets of $\beta$-lactams in virtually all bacteria are the high-molecular weight penicillin-binding proteins (PBPs) that catalyze the last cross-linking step of peptidoglycan polymerization. These enzymes are active-site serine D,D-transpeptidases that generate $4 \rightarrow 3$ cross-links connecting glycan strands. Mycobacteria contain additional targets for $\beta$ lactams since their peptidoglycan mainly (75-80\%) contains $3 \rightarrow 3$ cross-links formed by L,Dtranspeptidases. These enzymes are structurally unrelated to PBPs, contain an active-site cysteine residue, and use an acyl donor containing a stem tetrapeptide, in contrast to D,Dtranspeptidases, which use a pentapeptide. The essential tetrapeptide-containing substrate of the L,D-transpeptidases is formed by D,D-carboxypeptidases that cleave the D-Ala ${ }^{4}$-D-Ala ${ }^{5}$ bond of pentapeptide stems assembled in the cytoplasm. The latter enzymes represent a third class of targets for $\beta$-lactams since they belong to the active-serine PBP family. PBPs are potentially inactivated by all classes of $\beta$-lactams whereas L,D-transpeptidases are only inactivated by carbapenems. Other $\beta$-lactams only partially inactivate L,D-transpeptidases since formation of covalent adducts and their hydrolysis occur at similar slow velocities. However, this is not sufficient for resistance since inhibition of the D,D-carboxypeptidases indirectly inhibits the L,D-transpeptidases by blocking the supply of the tetrapeptide substrate. Intrinsic resistance to $\beta$-lactams in mycobacteria does not appear to result from inefficient inactivation of the targets, the PBPs and the L,D-transpeptidases, by these drugs. Instead, the mycomembrane limits access of $\beta$-lactams to the periplasm. The activity of the drugs is further limited by broad-spectrum class $C \beta$-lactamases. Imipenem (a carbapenem) and cefoxitin (a cephamycin) are part of the reference treatment of pulmonary infections due to Mycobacterium abscessus since these drugs are only slowly hydrolyzed by the $\beta$-lactamase $B_{\mathrm{Mab}}$. Among approved drugs, avibactam is the only $\beta$-lactamase inhibitor that efficiently inactivates $\mathrm{Bla}_{\mathrm{Mab}}$ in vitro. Avibactam also inactivates $\mathrm{Bla}_{\mathrm{Mab}}$ in human macrophages and in zebrafish embryos infected by $M$. abscessus. This inhibitor may potentially increase the panel of $\beta$-lactams useful to treat infections due to this bacterium, raising the possibility to use better tolerated and more efficient drugs in cystic fibrosis patients. 


\title{
Comparative analysis of systemic and local host-responses against MAP and MAH in an experimental goat model
}

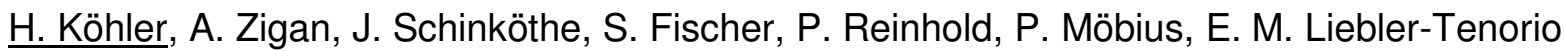

Institute of Molecular Pathogenesis, Friedrich-Loeffler-Institut, Jena, heike.koehler@fli.bund.de

Domestic ruminants are natural hosts for Mycobacterium (M.) avium-complex (MAC) infections. Paratuberculosis caused by $M$. avium subsp. paratuberculosis (MAP) is prevalent in cattle and goats, while M. avium subsp. hominissuis (MAH) infections are only incidentally observed. Experimental ruminant models of both infections allow insights into differences in host-pathogen interaction. This study aimed at examining the kinetics and intensity of systemic and local host responses of goats to MAP and MAH.

21 goat kids each were orally inoculated 10 times every 2-4 days with MAP or MAH, receiving total doses of $6,3 \times 10^{8}$ or $2,1 \times 10^{10}$ cfu per animal, respectively. Clinical symptoms, faecal shedding of the organisms, course of specific interferon-gamma (IFN- $\gamma$ ) and antibody responses as well as composition and specific activation of $\mathrm{T}$ cell subsets in blood were monitored regularly up to 13 months post inoculation (mpi) in inoculated animals and 10 agematched controls. At necropsy, which occurred at different time points pi, bacterial burden was culturally determined in intestinal tissues, intestinal lymph nodes (ILN) and in peripheral organs. Gene expression of immune modulatory cytokines (IFN- $\gamma$, IL-1 $\beta$, IL-10, IL-12p40, IL17 , IL-18, IL-23, TGF- $\beta$, TNF- $\alpha$ ) in organized gut associated lymphatic tissue (oGALT) and ILN was examined.

MAP-inoculated and control goats were clinically healthy throughout the experiment. In contrast, 9 of the MAH-inoculated goats developed progressive disease (fever, depression, weight loss) and died or were euthanized 2-3 mpi. The other goats had mild transient symptoms and were in good general condition when necropsied at $13 \mathrm{mpi}$. Already 7-10 weeks (w) pi, a strong antigen-induced IFN-y response was noted in MAP- and MAHinoculated animals. While the initial response was very high in the MAH-inoculated goats, it only gradually increased up to $4 \mathrm{mpi}$ in the MAP-inoculated animals. The proportion of $\mathrm{CD}^{+}{ }^{+} \mathrm{T}$ cells was up-regulated and that of $\gamma \delta \mathrm{TcR}^{+} \mathrm{T}$ cells down-regulated in $\mathrm{MAH}$-goats up to 6 mpi. Activation of $\mathrm{CD8}^{+} \mathrm{T}$ cells in blood was temporarily observed in both MAP and MAH inoculated groups. MAP-specific antibody titers started to increase about $3 \mathrm{mpi}$ and reached peak levels at $7 \mathrm{mpi}$. MAH-specific antibody titers were already measurable $2 \mathrm{mpi}$ but S/P\% values were considerably lower than in MAP-inoculated goats.

High to very high amounts of MAP were culturally isolated from intestinal mucosa, oGALT and ILN in animals necropsied 4 and $7 \mathrm{mpi}$, while low to moderate amounts were recovered at $13 \mathrm{mpi}$. Extra-intestinally, MAP was most often recovered from hepatic lymph nodes. Moderate to high amounts of MAH were recovered from intestinal mucosa, oGALT and ILN of the animals at 2-3 mpi. Massive dissemination to extra-intestinal tissues including liver, lung and spleen was also noted. At $13 \mathrm{mpi}$, MAH was almost eliminated and could only be isolated in low amounts from a few tissues of $7 / 10$ animals.

A differential regulation of the gene expression of IFN- - , IL-1 $\beta, I L-10, I L-12 p 40, I L-17$, IL-18, IL-23, TGF- $\beta$ and TNF- $\alpha$ was noted in the different tissues depending on mycobacterial species and time pi. The up-regulation of IL-17 in oGALT of MAH- and MAPinoculated animals necropsied 2-3, 4 and $7 \mathrm{mpi}$ in comparison to $13 \mathrm{mpi}$ was most prominant. At $13 \mathrm{mpi}$, IL-23 expression was increased in oGALT and ILN of MAP-inoculated goats compared to $\mathrm{MAH}$ - and control animals.

In conclusion, MAP and MAH interact differently with their natural host, the goat. Comparative studies will foster insights into protective mechanisms against MAC members. 


\section{Analysis of the virulence and immunogenicity of porcine and human M. avium ssp. hominissuis isolates in an experimental mouse model}

Nicolas Bruffaerts ${ }^{1}$, Christelle Vluggen ${ }^{1}$, Lucille Duytschaever ${ }^{2}$, Joseph Denoël ${ }^{3}$, Ruddy Wattiez $^{4}$, Jean-Jacques Letesson ${ }^{5}$, Vanessa Mathys ${ }^{1}$, Virginie Roupie ${ }^{2}$, David Fretin ${ }^{2}$, Claude Saegerman ${ }^{3}$ and Kris Huygen ${ }^{1}$

${ }^{1}$ WIV-ISP, ${ }^{2}$ CODA-CERVA, ${ }^{3}$ ULiège, ${ }^{4}$ UMons, ${ }^{5}$ UNamur

Nontuberculous mycobacteria of the Mycobacterium avium complex (MAC) are opportunistic pathogens of increasing concern for public health, due to their ability to cause lymphadenitis in children and systemic infections in immunocompromised patients. In pigs these bacteria can cause granulomatous lymphadenitis. Detailed comparison of human and swine MAC genotypes may shed more light on their zoonotic potential. Of ninety-nine human MAC isolates more than $95 \%$ were identified as $M$. avium ssp. hominissuis (Mah), and thirty-eight different genoypes were identified using multispacer sequence typing (MST). In $5 \%$ of suspected cases of submandibular granulomatous lymphadenitis in swine, found in Belgian slaughterhouses, ten different MST types of Mah were detected.

Using an experimental intranasal infection in BALB/c mice, we compared the virulence and immunogenicity of porcine and human isolates with shared genotype (MST 12, 22 and 91) or with specific human (MST 15) and porcine genotype (MST 103).

Isolates varied in virulence, with human/porcine genotype 22 and porcine genotype 12 showing higher bacterial numbers in lungs and more dissemination to spleen and liver than human/porcine genotype 91 and genotype 15. Human genotype 12 and genotype 103 showed an intermediate virulence. Mycobacteria specific cellular and humoral immune responses against a series of 37 recombinant $M$. avium proteins also showed pronounced variations.

In conclusion, intranasal infection of BALB/c mice may be a valuable experimental model for the development of tools for the immunosurveillance of Mah infection and the elucidation of its zoonotic potential.

Work supported partially by RT 12/5 LYMPHINDIC. 


\title{
Using whole genome sequencing to uncover the biology of NTM infection
}

\author{
Josephine Bryant $^{1 *}$, Dorothy Grogono ${ }^{2,3 *}$, Julian Parkhill ${ }^{1}, \underline{\text { R. Andres Floto }}^{2,3,4}$ \\ ${ }^{1}$ Wellcome Trust Sanger Institute, Hinxton UK \\ ${ }^{2}$ Cambridge Centre for Lung Infection, Papworth Hospital NHS Trust \\ ${ }^{3}$ Department of Medicine and ${ }^{4}$ Cambridge Institute for Medical Research, University of \\ Cambridge, UK \\ ${ }^{*}$ Contributed equally to this work
}

Mycobacterium abscessus is a rapidly emerging respiratory pathogen affecting $\sim 5-15 \%$ of individuals with Cystic Fibrosis around the world. It is inherently multi-drug resistant and challenging to treat, leads to accelerated decline in lung function and progressive lung damage, and is a contra-indication to lung transplantation in many centres. Prior to our work, infection with Mycobacterium abscessus was thought to be acquired by susceptible individuals independently from the environment. However, given the rise in infection rates worldwide, we examined whether person-to-person spread could be occurring.

Using whole genome sequencing and detailed epidemiological analysis of a cohort of patients attending the CF centre at Papworth Hospital, we found strong evidence for transmission from person to person. Our subsequent multi-national study has demonstrated the presence of a number of dominant global clones of $M$. abscessus with frequent personto-person transmission events occurring in CF centres around the world.

The presence of dominant circulating clones combined with evidence for transmission between patients suggests that Mycobacterium abscessus may be adapting to the lung environment. Deep sequencing of longitudinal isolates has allowed us to examine the changes in genetic diversity within a single individual over the course of chronic infection. We found that within-host variation correlates with bacterial burden and that both of these are reduced by effective antibiotic treatment. We observed phenotypic heterogeneity in antibiotic resistance and colony morphology which suggests that the development of within-patient variation can lead to clinically relevant changes. In addition to this we found evidence of convergent evolution in genes linked to virulence and of mutations conferring a hypermutator phenotype.

Our data provides the first evidence that Mycobacterium abscessus is frequently able to transmit from person-to-person and offers novel insights into population dynamics and withinpatient evolution of this organism. 


\section{Cystic fibrosis airways microbiota - from ecology to treatment}

\section{$\underline{\text { Kenneth Bruce }}$}

Institute of Pharmaceutical Science, King's College London, London SE1 9NH, United Kingdom; kenneth.bruce@kcl.ac.uk

Despite important therapeutic advances, mortality for the majority of individuals with cystic fibrosis is still due to respiratory failure resulting from damage triggered by airways infection. As such, it is important to detail and describe the roles of the microbes driving these infections. Over the past decade, culture-independent techniques have started to define the microbes - collectively the microbiota - in the lower airways of individuals with CF. For adults with cystic fibrosis, the airways microbiota has been shown typically to be highly complex with a phylogenetically-diverse range of bacterial species present. The species detected in microbiota studies include those such as Pseudomonas aeruginosa that have been regarded conventionally as pathogens in this context. Microbiota studies have also however detailed many species seldom if ever previously reported in the CF airways. In addition to anaerobes from the oral cavity, there is now increasing interest in understanding the role that NTM play in relation to the pathophysiology of CF airways disease progression. The focus of this presentation therefore is to contextualise NTM within the wider bacterial community of the CF airways microbiota. The impact of antibiotics on the CF airways microbiota will be discussed and future directions for research towards gaining therapeutic benefits for individuals with CF also identified. 
Poster 


\title{
Detection and immune characterization of Mycobacterium abscessus infection in cystic fibrosis patients
}

\author{
Nkwouano $\mathrm{V}^{1}$, Steindor $\mathrm{M}^{1}$, Mayatepek $\mathrm{E}^{1}$, Mackenzie $\mathrm{CR}^{2}$, Schramm $\mathrm{D}^{1}$, Jacobsen $\mathrm{M}^{1}$ \\ ${ }^{1}$ Department of General Pediatrics, Neonatology, and Pediatric Cardiology, University \\ Children's Hospital, Heinrich Heine University Duesseldorf, Germany; \\ vanesa.nkwouano@med.uni-duesseldorf.de \\ ${ }^{2}$ Institute of Medical Microbiology and Hospital Hygiene, Heinrich Heine University \\ Duesseldorf, Germany \\ Cystic fibrosis (CF) is a genetic disease caused by mutations of the Cystic Fibrosis \\ Transmembrane Conductance Regulator (CFTR) gene. CF patients suffer from multiple \\ infections with different pathogens, especially non-tuberculous mycobacteria [e.g. \\ Mycobacterium (M.) abscessus complex (MABSC)]. Specific immunological or PCR-based \\ tests for MABSC infection are not available. The aim of this study was to establish specific \\ tests for rapid detection of MABSC infections and to characterize T-cell immunity against \\ MABSC.
}

Whole blood collected from CF patients $(n=35)$ was re-stimulated in vitro using purified protein derivatives (PPD) of different mycobacteria (MABSC, M. tuberculosis, M. avium) and intracellular cytokine analysis was performed. Subpopulation marker (i.e. CD4, CCR7, CD45RA) as well as cytokine expression profiles (i.e. TNFa, IFNy, CD40L, IL-2) of mycobacteria specific $T$ cells were compared between CF patients with and without mycobacterial infections. For a subgroup of patients $(n=21)$, expectorated sputum was analyzed by mycobacterial culture and direct PCR based DNA strip test concomitantly.

Four patients with acute MABSC infections were positively tested by sputum culture and DNA strip test. These results were confirmed by MABSC PPD-induced T-cell immunity that also suggested previous mycobacterial infections in MABSC negative CF patients, as well as in a subgroup of CF patients not able to expectorate sputum. Quantification of CD40L/IL-2 double-positive T cells was optimal for detection of MABSC specific immunity and CD40L positive but IL-2 negative T cells qualified as candidate markers for acute MABSC infection in CF patients.

In conclusion, in vitro T-cell cytokine analysis in combination with PCR DNA strip tests enabled rapid diagnosis and characterization of MABSC infection, and may contribute to diagnosis especially where sputum samples are not available. 


\title{
Unusual outcome of an experimental infection of goat kids with Mycobacterium avium subsp. hominissuis
}

\author{
E. M. Liebler-Tenorio, J. Schinköthe, P. Möbius, S. Fischer, P. Reinhold, H. Köhler
}

Institute of Molecular Pathogenesis, Friedrich-Loeffler-Institut, Jena

Eisabeth.Liebler-Tenorio@fli.bund.de

Mycobacterium avium subsp. hominissuis (MAH) is one of 4 subspecies of $M$. avium. It is an opportunistic pathogen with a wide host spectrum including humans. Granulomatous lesions of the intestinal tract, especially intestinal lymph nodes and liver, but also systemic infections may occur. In the following, the findings after an experimental infection of goats are described.

Eighteen goat kids were orally inoculated (10 times, every 2-4 days) with a total dose of $10^{10}$ cfu MAH strain 09MA1289. Unexpectedly, 9 goats developed progressive disease (fever, depression, weight loss) and died or were euthanized in extremis 2-3 months post inoculation (mpi). The other goats had mild transient symptoms and were in good general condition when necropsied at $13 \mathrm{mpi}$. At necropsy, tissues were collected for histology and cultural isolation of mycobacteria. Lesions were characterized in HE-stained paraffin sections. MAH was labeled by immunohistochemistry (IHC).

MAH strain 09MA1289 used for inoculation was a porcine isolate with a multibanded IS 1245 RFLP pattern and the MIRU-VNTR genotype INMV 46 unique for Germany until now but found one time before for a French isolate originating also from pig (Randomski et al., 2010). Phenotypic and other genotypic characteristics of this strain (presence of ISMpa1 and a selection of specific GPL genes) were commonly found in other MAH strains in Germany.

Goats necropsied with severe clinical signs were in bad nutritional condition. There was extensive ulceration of organized gut associated lymphatic tissue (oGALT) and necrosis of intestinal lymph nodes (ILN). Ulcerations were associated with granulomatous infiltrates composed of many lymphocytes, small groups of epitheloid cells and variable numbers of neutrophils. The confluent granulomas in ILN were delineated by granulomatous infiltrates. Granulomatous infiltrates were also detected in multiple other sites. MAH and mycobacterial material was detected in variable amounts and MAH was cultured from oGALT, ILN and numerous other sites of the 9 goats.

All of the apparently healthy goats necropsied at $13 \mathrm{mpi}$ had severely enlarged ILN with large granulomas and several had multiple small granulomas in oGALT. Mycobacterial material was regularly detected in ILN and occasionally in ileal Peyer's patches. MAH was cultured from ILN of 4 goats.

In conclusion, goats have to be included in the list of species susceptible to MAH infection. After oral exposure, lesions dominate in oGALT and ILN, but there is also systemic spread. Even in apparently healthy animals, lesions were regularly found and may contain viable $\mathrm{MAH}$. The different outcome of the MAH infection in a homogenous group of animals under the same experimental conditions and the severity of lesions in young animals are unusual. 


\title{
Morphological characterization of granulomas in goats experimentally infected with Mycobacterium avium subsp. hominissuis
}

\author{
J. Schinköthe, H. Köhler, E. M. Liebler-Tenorio
}

Institute of Molecular Pathogenesis, Friedrich-Loeffler-Institut, Jena jan.schinkoethe@fli.bund.de

Mycobacterium avium subsp. hominissuis (MAH), a non-tuberculous mycobacterium (NTM), causes chronic granulomatous diseases in humans and animals. This study describes the morphology of granulomas induced by MAH and compares them with those seen in $M$. tuberculosis complex (MTC) infection.

Granulomas were detected in organized gut associated lymphatic tissues (oGALT) and intestinal lymph nodes (ILN) of goats experimentally infected with MAH when they were necropsied 2-3 months post inoculation (mpi) and $13 \mathrm{mpi}$. According to a modified scheme from Wangoo et al. (2005) granulomas were characterized in HE-stained paraffin sections as (1) stage 1: initial infiltrate (2) stage 2: small, solid with minimal necrosis, (3) stage 3: monocentric with central necrosis and mineralization (4) stage 4: multicentric with central necrosis and mineralization and (5) stage 5: predominantly fibrotic organization. Macrophages, T-cell subsets, B-lymphocytes, plasma cells and MHC-II positive cells were labeled in frozen sections by immunohistochemistry (IHC) to determine the cellular composition of granulomas. MAH was stained in frozen sections with the Kinyoun method.

At 2-3 mpi, granulomas of stage 1, 2, 3 and 4 were seen in ILN, but not in oGALT. Granulomas of stages 3 and 4 predominated. They were characterized by extensive caseous necrosis with minimal mineralization surrounded by moderate numbers of epitheloid cells, CD4+ cells, few macrophages, single CD8+ cells and scattered $ү \delta$ T-cells. Single MAH were seen in few epitheloid cells in all granuloma stages and in variable quantities in areas of necrosis in type 3 and 4 granulomas. Many MAH were seen in epitheloid cells and necrosis of type 3 and 4 granulomas in one goat.

At $13 \mathrm{mpi}$, granulomas were found both in oGALT and ILN. In addition to granulomas of stage 1 to 4, stage 5 granulomas were present in ILN. Stage 3 granulomas where the central necrosis and mineralization was surrounded by many epitheloid cells, few multinucleated giant cells and CD4+ T-cells followed by a layer of tertiary lymphoid tissue dominated in oGALT. Large multicentric granulomas with marked fibrotic organization and little cellular infiltrate (stage 4 and 5 granulomas) were frequent in ILN. Single MAH were seen in scattered epitheloid cells and areas of necrosis in few granulomas only.

In conclusion, MAH elicits granulomas highly similar to those in MTC infections and might serve as large animal model for the pathogenesis of these granulomas. 


\title{
Distribution of specific glycopeptidolipids (GPLs) within subspecies of Mycobacterium avium
}

\author{
Petra Möbius ${ }^{1}$, Janina Kolb², Doris Hillemann², Elvira Richter ${ }^{2}$, Heike Köhler ${ }^{1}$ \\ ${ }^{1}$ Friedrich-Loeffler-Institut (Federal Research Institute for Animal Health), Institute of \\ Molecular Pathogenesis, National Reference Center for Paratuberculosis, Jena, Germany; \\ petra.moebius@fli.bund.de \\ ${ }^{2}$ Research Center Borstel, National Reference Center for Mycobacteria, Borstel, Germany
}

Members of Mycobacterium avium (M. avium) belong to the direct and indirect human environment; they have been found in soils and waters worldwide. M. avium subsp. hominissuis (MAH) is an opportunistic environmental pathogen responsible for infections in humans, swine and other mammals. M. avium subsp. avium (MAA) and M. avium subsp. silvaticum (MAS) are the causative agents of avian tuberculosis; $M$. avium subsp. paratuberculosis (MAP) causes Johne's disease in ruminants. The interaction of M. avium members with their environment is crucial in the determination of their pathogenicity. Cell wall glycolipids (GPLs) are discussed to contribute to the virulence of $M$. avium. Different genes involved in the synthesis of GPLs would be expected to modify therefore indirectly the interaction of the bacteria with their hosts.

The aim of this study was to get deeper insight into the distribution of genes involved in the synthesis of GPLs among subspecies of $M$. avium and among strains of subsp. paratuberculosis. Therefore, $M$. avium isolates of different origin were screened for the presence of six selected genes of the GPL cluster.

Altogether 76 M. avium isolates belonging to MAA $(n=10)$, MAH $(n=25)$, and MAP $(n=40)$ originating from different hosts and some from the environment were investigated including the reference strains ATCC 25291 (MAA), ATCC 19698 (MAP), DSM 44175 (MAS), and additionally the ATCC 13950 ( $M$. intracellulare). The isolates were tested for the presence of selected nonspecific (ns)GPL genes gtfA, rtfA, and $m t f C$ as well as serotype-specific (ss)GPL genes: $m d h t A$, merA, mtfF by PCR using primers from Johansen et al. (2009). All M. avium isolates had been characterized before by MIRU-VNTR, RFLP and MLST genotyping.

The selected ssGPL genes $m d h t A$, merA, and mtfF were detected in most examined strains with the exception of two MAH isolates and M. intracellulare. All MAH and MAA strains, the reference strains of MAS and $M$. intracellulare, and 16 MAP strains (comprising 15 MAP-C strains and one MAP-S strain) revealed the three nsGPL genes gtfA, rtfA, and mtfC. In 24 MAP isolates no nsGPL gene sequences could be found. One bovine MAP strain lacked only the gtfA gene sequence. Furthermore, there was no association between presence or absence of the three nsGPL genes in MAP and a specific MAP genotype. The presence of $g t f A, r t f A$, and $m t f C$ in more than one third of the studied MAP strains and in the MAS reference strain is in contrast to previous results of Eckstein et al. (2003). The importance of this division of MAP strains into two groups with or without selected nsGPL genes regarding specific membrane characteristics and different interactions with their hosts has to be clarified in further investigations.

References:

Eckstein et al. (2003) Microbiology 149, 2797-2807;

Johansen et al. (2009) BMC Microbiology 9:159 doi:10.1186/1471-2180-9-159. 


\title{
MLSA-Typing of non-tuberculous mycobacteria isolated from bovine lymph nodes in Switzerland
}

\author{
Olivier Dietz $^{1}$, Patricia Landolt ${ }^{1}$, Ute Friedel ${ }^{1}$, Max M. Wittenbrink ${ }^{1}$ \\ ${ }^{1}$ Institute of Veterinary Bacteriology, Vetsuisse Faculty University of Zurich, Switzerland, \\ olivier.dietz@vetbakt.uzh.ch
}

With more and more studies revealing that non-tuberculous Mycobacteria (NTM) cause severe disease in humans and animals, NTM finally start to emerge from the enormous shadow of M. tuberculosis. However, despite their increased recognition as causative agents of numerous skin and soft tissue infections, lung diseases and visceral or disseminated diseases, still surprisingly little is known about NTM prevalence in Switzerland.

In order to evaluate the spectrum of NTM species infecting Swiss cattle, we investigated 63 M. tuberculosis complex (MTBC) negative Mycobacteria strains isolated from bovine lymph nodes. By multi locus sequence analysis (MLSA) based on 16S rRNA, rpoB, hsp65 and 16S$23 S$ internal transcribed spacer (ITS), the species of 53 strains could be identified successfully. While the predominant species was $M$. avium subsp. hominissuis (29\%), multiple strains were also isolated of $M$. nonchromogenicum (11\%), M. neoaurum (8\%), $M$. kansasii (6\%), M. monacense (5\%), M. xenopi (5\%) and M. vaccae (3\%). Additionally eleven NTM species were isolated only once from the investigated lymph nodes. Of those particularly interesting are $M$. abscessus and $M$. engbaekii which to our knowledge both have not been detected in cattle before.

While some studies identified NTM as important pathogens, others revealed that certain NTM can further interfere with tuberculosis (TB) diagnosis. The most commonly used tools for TB testing, tuberculin skin tests and Interferon-gamma release assays (IGRA), both measure reactivity of the immune system against the $M$. tuberculosis antigens ESAT- 6 and CFP-10 whose genes are located within the so called region of difference 1 (RD1). Since RD1 was also found in the genome of several NTM including M. marinum, M. szulgai and $M$. kansasii, an infection with the respective agent can result in false-positive TB-diagnosis. In this context our identification of four M. kansasii strains is of utmost interest. With this finding the paradigm that $M$. kansasii infections do not exist in Swiss cattle could finally be disproved. In order to investigate if additional NTM strains do also possess the RD1 region, esat- 6 and cfp-10 specific PCRs were performed. While most of the other strains were RD1 negative, our study revealed that $M$. gordonae does possess the $c f p-10$ gene.

Summarized our results not only show for the first time that Swiss cattle are infected by various NTM species, they also indicate that some of these infections interfere with tuberculosis diagnosis. Therefore further investigation of NTM prevalence in Swiss cattle is crucial, also regarding an efficient TB control. 


\title{
Non-tuberculous mycobacterial infections of veterinary relevance
}

\begin{abstract}
Franck Biet $^{1}$ and Maria-Laura Boschiroli ${ }^{2}$
${ }^{1}$ Institut National de la Recherche Agronomique, Université de Tours, Unité Mixte de Recherches 1282, Infectiologie et Santé Publique, INRA Centre Val de Loire 37380 Nouzilly. ${ }^{2}$ University Paris-Est, Anses, Laboratory for animal health, Bovine tuberculosis national reference laboratory,Bacterial Zoonoses Unit, 23, avenue du Général de Gaulle, 94706 Maisons-Alfort cedex, France.

1Franck.Biet@tours.inra.fr

Mycobacteria play an important role in human and animal health fields. Here we examined the place of NonTuberculous Mycobacteria (NTM) infections in the veterinary context. In this review relevant aspects regarding a reference laboratory experience and the literature review are presented. Importance is given as regards productivity lose per se but also to economic losses due to misdiagnosis with bovine tuberculosis and paratuberculosis. The importance of the role played by NTM is variable depending on the part of the world, ecology, husbandry, extent of surveillance programs and bovine tuberculosis and paratuberculosis prevalence. The role in animal disease of the most relevant NTM are summarized with a focus on Mycobacterium avium subsp. paratuberculosis which occupies a particularly important position, being the causative agent of paratuberculosis, a disease responsible of high economic consequences for ruminant livestock.
\end{abstract}




\title{
Comparison in the mouse model of several potential vaccine targets against Mycobacterium abscessus in order to obtain protection in the context of cystic fibrosis
}

\author{
Vincent Le Moigne ${ }^{1}$, Benoît Barteau ${ }^{2}$, Bruno Pitard ${ }^{2}$, Nigou Jérôme ${ }^{4}$, Fabienne Girard- \\ Misguich ${ }^{1}$, Stéphane Canaan ${ }^{3}$, Jean-Louis Gaillard ${ }^{1}$; Jean-Louis Herrmann ${ }^{1}$ \\ ${ }^{1}$ Université de Versailles-St-Quentin-en-Yvelines, Montigny-le-Bretonneux, France \\ vincent.le-moigne@uvsq.fr \\ ${ }^{2}$ UMR 915/INSERM, Nantes, France ; \\ ${ }^{3}$ CNRS, UMR 7282-EIPL, Marseille, France ; \\ ${ }^{4}$ CNRS, IPBS, Toulouse, France .
}

In the fight against multi-resistant bacteria to antibiotics, vaccinal strategies represent a therapeutic possibility. Mycobacterium abscessus, a currently emerging pathogen belonging to the group of rapidly growing mycobacteria (RGM), is one of the most resistant bacteria responsible for respiratory and cutaneomucous infections. Cystic fibrosis patients represent one of the most susceptible population of patients. In order to obtain protection against this bacterium, various antigens were tested using different immunization routes. Virulence factors such as PLC (phospholipase C) or MgtC have been tested, an enzyme from the family of Nitrilases (MAB_2545c) and a more complex fraction from surface extract selected specifically for its ability to activate the response innate via TLR2. We studied the immune response and protection in mice infected intravenously (IV) or by aerosol route with $M$. abscessus after DNA or protein immunization of these $M$. abscessus antigens. The mice were either wild-type mice or mice with the most common mutation observed in the context of cystic fibrosis $(\Delta \mathrm{F} 508)$. The three monovalent antigens show all, to varying degrees (depending on the infectious mode, the immunization method chosen and the type of mouse studied), a significant protective effect in infected mice. Generally speaking, the elimination of bacteria is faster when the infection occurs by the aerosol route, and it is more efficient for $\Delta \mathrm{F} 508$ mice as for their wild-type littermates (FVB). DNA vaccination is similar to the protein vaccination for PLC antigen but does not work for the Nitrilase antigen while the latter immunization as purified protein is very efficient. Regarding the TLR2-activating complex fraction, there is no protection after infection by the aerosol route but it is effective after IV infection, curiously only on certain organs tested (liver and lungs).

In conclusion, several proteic antigens, particularly one of them, the Nitrilase MAB_2545c, are good vaccine candidates against $M$. abscessus in the context of cystic fibrosis. 


\title{
Detection and immune characterization of Mycobacterium abscessus infection in Cystic Fibrosis patients
}

\author{
Nkwouano $\mathrm{V}^{1}$, Steindor $\mathrm{M}^{1}$, Mayatepek $\mathrm{E}^{1}$, Mackenzie $\mathrm{CR}^{2}$, Schramm $\mathrm{D}^{1}$, Jacobsen $\mathrm{M}^{1}$ \\ ${ }^{1}$ Department of General Pediatrics, Neonatology, and Pediatric Cardiology, University \\ Children's Hospital, Heinrich Heine University Duesseldorf, Germany; \\ vanesa.nkwouano@med.uni-duesseldorf.de \\ ${ }^{2}$ Institute of Medical Microbiology and Hospital Hygiene, Heinrich Heine University \\ Duesseldorf, Germany
}

Cystic fibrosis (CF) is a genetic disease caused by mutations of the Cystic Fibrosis Transmembrane Conductance Regulator (CFTR) gene. CF patients suffer from multiple infections with different pathogens, especially non-tuberculous mycobacteria [e.g. Mycobacterium (M.) abscessus complex (MABSC)]. Specific immunological or PCR-based tests for MABSC infection are not available. The aim of this study was to establish specific tests for rapid detection of MABSC infections and to characterize T-cell immunity against MABSC.

Whole blood collected from CF patients $(\mathrm{n}=35)$ was re-stimulated in vitro using purified protein derivatives (PPD) of different mycobacteria (MABSC, M. tuberculosis, M. avium) and intracellular cytokine analysis was performed. Subpopulation marker (i.e. CD4, CCR7, CD45RA) as well as cytokine expression profiles (i.e. TNFa, IFNy, CD40L, IL-2) of mycobacteria specific $T$ cells were compared between CF patients with and without mycobacterial infections. For a subgroup of patients $(n=21)$, expectorated sputum was analyzed by mycobacterial culture and direct PCR based DNA strip test concomitantly.

Four patients with acute MABSC infections were positively tested by sputum culture and DNA strip test. These results were confirmed by MABSC PPD-induced T-cell immunity that also suggested previous mycobacterial infections in MABSC negative CF patients, as well as in a subgroup of CF patients not able to expectorate sputum. Quantification of CD40L/IL-2 double-positive $T$ cells was optimal for detection of MABSC specific immunity and CD40L positive but IL-2 negative T cells qualified as candidate markers for acute MABSC infection in CF patients.

In conclusion, in vitro T-cell cytokine analysis in combination with PCR DNA strip tests enabled rapid diagnosis and characterization of MABSC infection, and may contribute to diagnosis especially where sputum samples are not available. 


\title{
Development of new anti-mycobacterial agents against Mycobacterium abscessus infections
}

\author{
Faustine Dubar $^{1}$, Christian Dupont ${ }^{2,3}$, Yann Guérardel ${ }^{1}$, Jean-Louis Hermann ${ }^{4}$, Laurent \\ Kremer $^{2,3}$, Christophe Biot ${ }^{1}$. \\ ${ }^{1}$ Unité de Glycobiologie Structurale et Fonctionnelle (UGSF - UMR 8576) de l'Université Lille \\ 1, faustine.dubar@univ-lille1.fr
}

${ }^{2}$ Centre d'étude des Pathogènes pour la Biotechnologie et la Santé (CPBS Fre 3689)/ CNRS, 1919 route de Mende, 34293 Montpellier, France

${ }^{3}$ Université de Montpellier

${ }^{4}$ EA 3647 EPIM Université de Versaille Saint Quentin (UVSQ)

Mycobacterium abscessus is an emerging human pathogen responsible of bronchopulmonary infections in patients with cystic fibrosis (CF).It is considered as the most virulent and drug-resistant rapid-growing mycobacterial species. In CF patients, the presence of these mycobacteria is a contraindication to lung transplantation. This pathogen has two morphotypes: a smooth (S) avirulent variant characterized by the presence of glycopeptidolipids (GPL) and a rough (R) virulent variant, devoid of GPL surface expression. Recently, we developed a zebrafish model to study the chronology and physiopathology of $M$. abscessus infections thanks to the optical transparency of the embryos wich allowed monitoring the evolution of the infectious process in real time. This model was also used to evaluate the therapeutic activity of antibiotics in an in vivo context.

With the aim to identify new compounds active against $M$. abscessus, we screened a chemical library against whole bacteria. In vitro drug assessments were performed following the recommendations of the Clinical and Laboratory Standards Institute and activity was further confirmed in both liquid and solid media against a panel of $M$. abscessus clinical isolates from CF and non-CF patients. A route of chemical synthesis was also established to produce large amounts of material required to evaluate its therapeutic activity in pre-clinical studies in the zebrafish model of infection.

One compound, FMD-5 was identified as being very active against the $M$. abscessus reference strain with an MIC $\leq 0.20$ microM. Similar values were obtained against different clinical strains ( $S$ and $R$ ) exhibiting different profiles of susceptibility to imipenem or amikacin. Importantly, our preliminary results suggest that FMD-5 has potent in vivo activity in infected zefrafish. Exposure to compound was associated to i) a decrease of the pathophysiological symptoms and particularly the infection foci within the central nervous system, and ii) an increase survival of the infected embryos.

We identified a scaffold for a new anti-infective agent against $M$. abscessus. With the aim to better define the pharmacophore, a structure-activity relationships study has been initiated. Further studies will also be dedicated to address the antimycobacterial activity of the compounds in cftr- morphants, used to mimick a CF-like microenvironment. 


\title{
LOOXSTER ${ }^{\circledR}$ technology: overcoming obstacles in enrichment of mycobacteria from whole blood
}

\author{
$\underline{\text { Ute Hofmann }^{1}}$, Sönke Andres ${ }^{2}$, Elvira Richter ${ }^{2}$, André Reinhardt ${ }^{1}$, Dirk Osterloh ${ }^{1}$ \\ ${ }^{1}$ Analytik Jena AG, Konrad-Zuse-Straße 1, 07745 Jena, Germany \\ ute.hofmann@analytik-jena.de \\ ${ }^{2}$ Forschungszentrum Borstel, Nationales Referenzzentrum, Parkallee 1-40, 23845 Borstel, \\ Germany
}

Disseminated mycobacterial infections are serious diseases with a fatal outcome unless detected and treated fast. Compared to pulmonary manifestations they do occur only in immunocompromised patients, e.g. in patients with HIV/AIDS. Thus the detection of mycobacteria in immunocompromised patients often requires more laborious diagnostic methods than smear microscopy or cultivation of respiratory samples on standard media. Mycobacterial blood culture systems, such as MYCO/F LYTIC or BacT/ALERT MB, are the usual methods for the detection of mycobacteria in whole blood. Culturing techniques require several weeks to identify mycobacterial growth. Moreover, non-growing mycobacteria like $M$. genavense or $M$. tilburgii, which are mainly detected in immunocompromised patients, are not readily detectable with these methods. A rapid and culture independent identification technology, e.g. DNA enrichment procedure and a subsequent nucleic acid amplification test (NAAT), is needed urgently. New pre-analytic approaches aim to increase the sensitivity of NAATs through enrichment of microbial DNA. Cytosine methylation and frequency of nonmethylated $\mathrm{CpG}$ dinucleotides are genomic properties varying greatly between mammals and bacteria. Analytik Jena's LOOXSTER ${ }^{\circledR}$ uses the specific affinity of the CXXC zinc finger 1 domain to non-methylated $\mathrm{CpG}$ dinucleotides for powerful bacterial and fungal nucleic acid enrichment. Due to the removal of more than $95 \%$ of eukaryotic background DNA large sample sizes become accessible to analytical and preparative downstream applications, that is even more important when detecting very low microbial counts.

Within this study a manual workflow consisting of the PureProve ${ }^{\circledR}$ Blood \& Tissue DNA Maxi Kit for total DNA extraction followed by the enabling technology of the LOOXSTER ${ }^{\circledR}$ Enrichment Kit was used to extract and enrich M. fortuitum DNA from spiked blood samples. An in-house Real-time PCR method targeting an Internal Transcribed Spacer fragment and subsequent Sanger Sequencing was performed to verify the origin of the enriched DNA. The blood was spiked with different $\mathrm{cfu} / \mathrm{ml}$ of the bacterium; PCR was performed before and after the enrichment.

DNA of $M$. fortuitum could be detected in all samples independent on the cfu/ml after the enrichment with the LOOXSTER ${ }^{\circledR}$ Enrichment Kit. In contrast, the DNA could only be detected in the DNA extracts that have not been enriched by the technology when the blood sample was spiked with high amounts of bacteria. The results show that the workflow consisting of the PureProve ${ }^{\circledR}$ Blood \& Tissue DNA Maxi Kit for total DNA extraction followed by the enabling technology of the LOOXSTER ${ }^{\circledR}$ Enrichment Kit is suitable for the detection of mycobacterial DNA in spiked blood samples. 


\title{
Multi-tiered map containing both the coding and regulatory architecture of the Mycobacterium abscessus genome
}

\author{
Brendan Loftus, Aleksandra Miranda Caso Luengo*, Patrick Staunton*, Adam Dinan, \\ Amanda Lohan and Pin Tong
}

*Both authors contributed equally to the work

School of Medicine and Conway Institute, University College Dublin, Ireland

Contact: brendan.loftus@ucd.ie

Mycobacterium abscessus is a rapidly growing mycobacterium (RGM) that is both intrinsically highly antibiotic resistant and an important CF pathogen. The $M$. abscessus genome shares notable features with with slow growing mycobacteria including important virulence related genes while harboring notable differences at the genic level as a result of lateral gene transfer. Evolution of regulatory elements occurs at a more rapid rate however and a genome wide identification of regulatory elements that control the transcriptome and proteome has yet to be carried out in $M$. abscesssus. Understanding the extent to which these regulatory features are unique to $M$. abscessus or are shared across mycobacteria will shed important light on the biology of $M$. abscessus and its survival strategies within the host. Therefore we generated a multi-tiered omics map of the $M$. abscessus genome encompassing a transcriptional start site (TSS) map, combined with RNA-seq to identify genome wide RNA elements within the genome. In addition we have generated ribosome profiling and LC-MS proteomics data to identify which elements are coding (Fig 1). Together these provide a valuable genomic resource to understand the biology of $M$. abscessus.

Our analysis has identified 48 small un-annotated genes, many of which are highly expressed and a number of which are secreted. We identify numerous genes regulated by 5'UTR regulatory elements including leader peptides and putative riboswitches. We identify a small set of regulatory RNAs conserved across mycobacteria including those essential for growth and predict their mechanism of action. Finally we identify genes that appear to be partially translated offering a mechanism by which they can have independently both essential and non-essential domains for growth (Fig. 1).

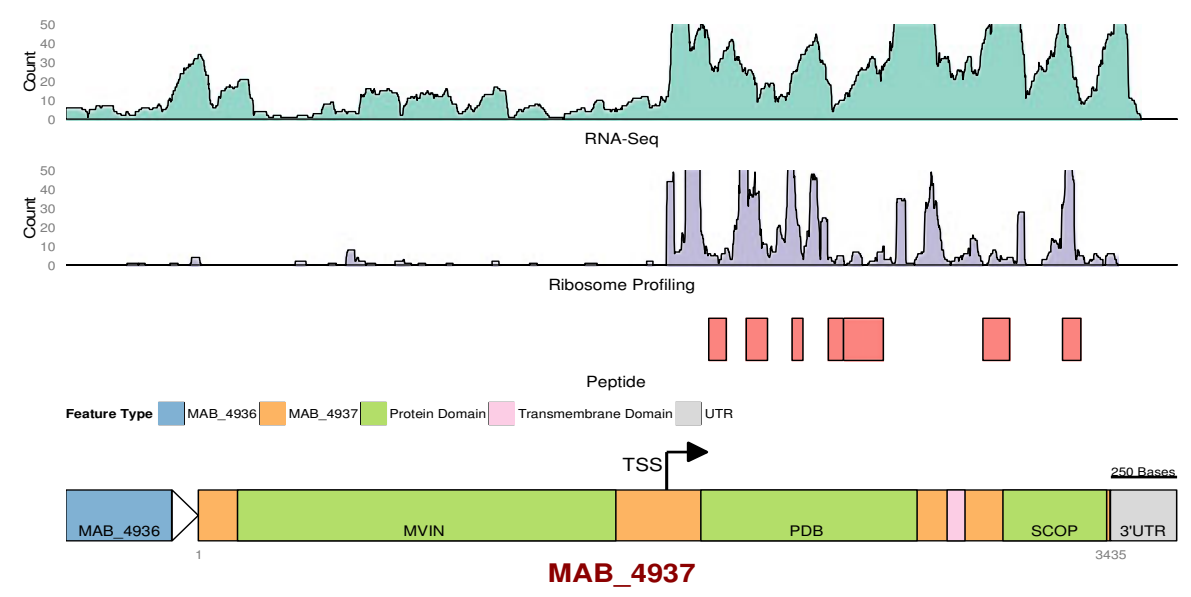


List of
Participants 
Dr. Frank Ahrens

Altonaer Kinderkrankenhaus von 1859

Frank.Ahrens@kinderkrankenhaus.net

Prof. Dr. Michel Arthur

INSERM UPMC UPD

michel.arthur@crc.jussieu.fr

Dr. Olaf Bartsch

Insmed GmbH

Olaf.Bartsch@Insmed.com

Hannelore Bax

Erasmus Medical Center Rotterdam

h.bax@erasmusmc.nl

Dr. Franck Biet

INRA Tours

Franck.biet@tours.inra.fr

Prof. Dr. Patrick Brennan

Colorado State University

patrick.brennan@colostate.edu

Dr. Kenneth Bruce

King's College London

kenneth.bruce@kcl.ac.uk

Prof. Dr. Delphi Chatterjee

Colorado State University

delphi.chatterjee@colostate.edu

Dr. Caroline Demangel

Institut Pasteur Paris

demangel@pasteur.fr

Dr. Olivier Dietz

Institue of Veterinary Bacteriology

Vetsuisse Faculty University of Zurich

olivier.dietz@vetbakt.uzh.ch

Dr. Faustine DUBAR

Université Lille 1

faustine.dubar@univ-lille1.fr

Dr. Katarzyna Duda

Research Center Borstel

kduda@fz-borstel.de

Prof. Dr. Torsten Eckstein

Colorado State University

eckstein@mail.colostate.edu
Prof. Dr. Stefan Ehlers

Research Center Borstel

sehlers@fz-borstel.de

Isobel Everall

Wellcome Trust Sanger Institute

ie3@sanger.ac.uk

Dr. Fabienne Misguich

Université Versailles Saint Quentin-en-

Yvelienes UVSQ

fabienne.girard-misguich@uvsq.fr

Prof. Dr. Andres Floto

University of Cambridge

arf27@cam.ac.uk

Dariimaa Ganbat

Research Center Borstel

dganbat@fz-borstel.de

Dr. Nicolas Gisch

Research Center Borstel

ngisch@fz-borstel.de

Prof. Dr. Ralph Goethe

Tierärztliche Hochschule Hannover

ralph.goethe@tiho-hannover.de

Prof. Dr. Thomas Gutsmann

Research Center Borstel

tgutsmann@fz-borstel.de

Prof. Dr. Jean-Louis Herrmann

UMR1173 Versailles St Quentin University jean-louis.herrmann@rpc.aphp.fr

Dr. Christian Herzmann

Research Center Borstel.de

cherzmann@fz-borstel.de

Dr. Wouter Hoefsloot

RadboudUMC / UCCZ Dekkerswald

wouter.hoefsloot@radboudumc.nl

Dr. Ute Hofmann Analytik Jena AG

ute.hofmann@analytik-jena.de

Dr. Christoph Hölscher

Research Center Borstel

choelscher@fz-borstel.de 
Prof. Dr. Otto Holst

Research Center Borstel

oholst@fz-borstel.de

Dr. Kris Huygen

Scientific Institute of Public Health (Belgium)

kris.huygen@wiv-isp.be

Dr. Heike Köhler

Friedrich-Loeffler-Institut

Heike.Koehler@fli.bund.de

Dr. Laurent Kremer

CNRS - University of Montpellier

laurent.kremer@cpbs.cnrs.fr

Prof. Dr. Christoph Lange

Research Center Borstel

clange@fz-borstel.de

Dr. Vincent le Moigne

INSERM U1173 - Université de Versailles-St-

Quentin-en-Yvelines

vincentlemoigne@uvsq.fr

Dr. Astrid Lewin

Robert Koch Institute Berlin

LewinA@rki.de

Prof. Dr. Elisabeth Liebler-Tenorio

Friedrich-Loeffler-Institut

Elisabeth.Liebler-Tenorio@fli.bund.de

Prof. Dr. Brendan Loftus

UCD

brendan.loftus@ucd.ie

Dr. Sven Malm

Research Center Borstel

smalm@fz-borstel.de

Prof. Dr. Liza Micioni

Insmed GmbH

Liza.Micioni@insmed.com

Dr. Petra Möbius

Friedrich-Loeffler-Institut

petra.moebius@fli.bund.de

Dr. Sven Müller-Loennies

Research Center Borstel

sml@fz-borstel.de
Prof. Dr. Stefan Niemann

Research Center Borstel

sniemann@fz-borstel.de

Vanesa Nkwouano

University Children's Hospital Duesseldorf

vanesa.nkwouano@med.uni-duesseldorf.de

Prof. Dr. Walter Oelemann

Instituto de Microbiologia

Universidade Federal do Rio de Janeiro (UFRJ)

Rio de Janeiro, Brazil

oelemann@micro.ufrj.br

Ioana Diana Olaru

Research Center Borstel

iolaru@fz-borstel.de

Dr. Kenneth Olivier

National Heart, Lung, and Blood Institute

kenneth.olivier@nih.gov

Prof. Dr. Diane Ordway

Colorado State University

D.Ordway@colostate.edu

Priv. Doz. Dr. Norbert Reiling

Research Center Borstel

nreiling@fz-borstel.de

Daniela Rodriguez

University of Cambridge

dr439@cam.ac.uk

Andrea Sanchini

Robert Koch Institute

sanchinia@rki.de

Dr. Hubert Schaefer

Robert Koch Institute

Schaeferh@RKI.de

Prof. Dr. Ulrich Schaible

Research Center Borstel

uschaible@fz-borstel.de

Jan Schinköthe

Friedrich-Loeffler-Institut (FLI)

jan.schinkoethe@fli.bund.de 
Dr. Bianca Schneider Research Center Borstel

bschneider@fz-borstel.de

Dr. Dominik Schwudke Research Center Borstel dschwudke@fz-borstel.de

Prof. Dr. Jakko van Ingen

Radboud University Medical Center vaningen.jakko@gmail.com

Dr. Nicolas Veziris

Centre National de Référence des Mycobactéries

nicolas.veziris@upmc.fr

Prof. Dr. Dirk Wagner

University of Freiburg

dirk.wagner@uniklinik-freiburg.de
Prof. Dr. Richard Wallace

UT Health Northeast

richard.wallace@uthct.edu

Dr. Nasstasja Wassilew

Research Center Borstel,

Medical Department/Clinical Infectious

Diseases

nwassilew@fz-borstel.de

Dr. Nicole Zehethofer

Research Center Borstel

nzehethofer@fz-borstel.de

Sanne Zweijpfenning

Radboud university

sannezweijpfenning@gmail.com 


\section{The Campus}

\section{Research Center Borstel}

Manor House

Parkallee 1

D-23845 Borstel/Sülfeld +49 $4537188-0$

Important phone numbers:

Otto Holst

Office: +49 45371884720

Cell phone

U. Schaible

Office: +49 45371882090

Secretary Mrs Passarger

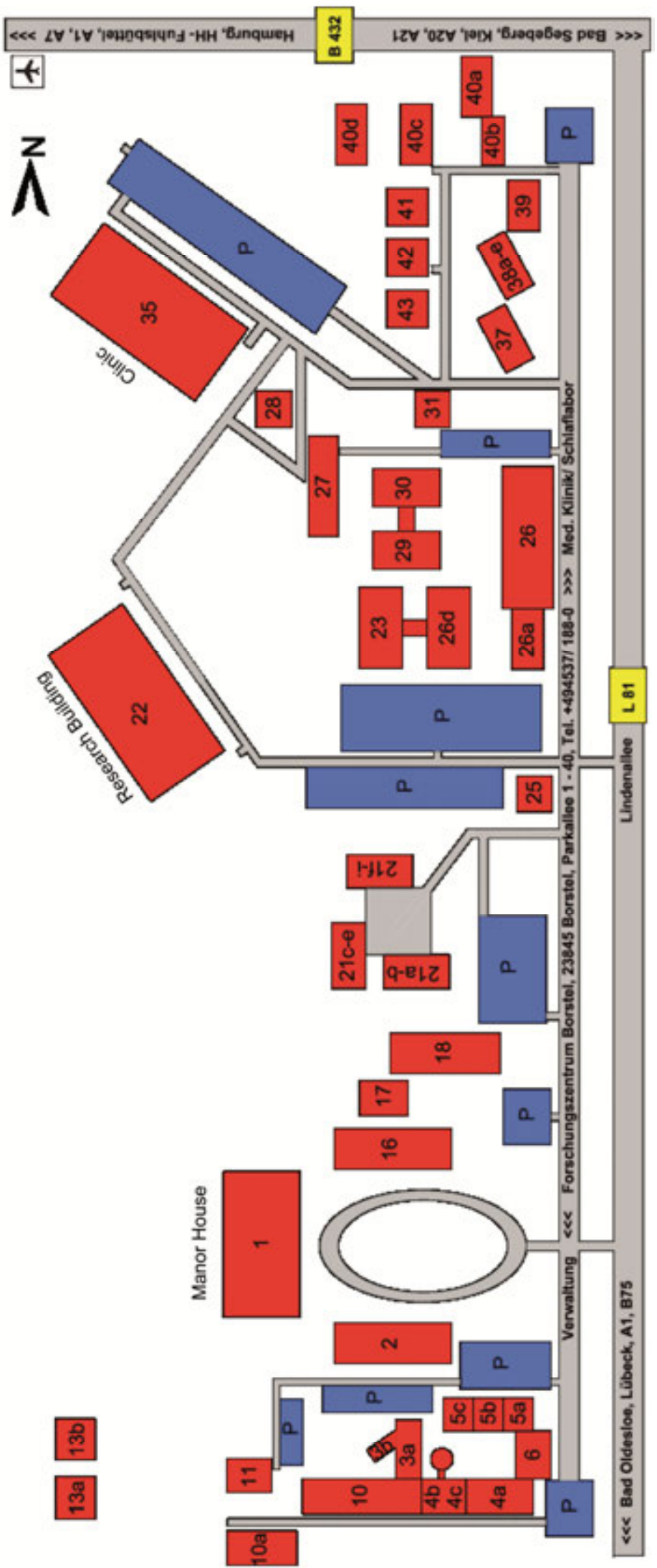

